Article

\title{
Palladium-catalyzed enantioselective linear allylic alkylation of vinyl benzoxazinanones: An inner-sphere mechanism
}

\author{
Kai Wang a,b, Binli Wang b,c, Xianghui Liu a,b, Hongjun Fan c,*, Yan Liu a,\#, Can Li a,\$ \\ a State Key Laboratory of Catalysis, Dalian Institute of Chemical Physics, Chinese Academy of Sciences, Dalian 116023, Liaoning, China \\ b University of Chinese Academy of Sciences, Beijing 100049, China \\ ' State Key Laboratory of Molecular Reaction Dynamics, Dalian Institute of Chemical Physics, Chinese Academy of Sciences, Dalian 116023, Liaoning, China
}

\section{A R T I C L E I N F O}

\section{Article history:}

Received 28 October 2020

Accepted 15 December 2020

Available online 5 March 2021

\section{Keywords:}

Pd catalysis

Asymmetric catalysis

Allylic alkylation

Vinyl benzoxazinanones

Inner-sphere mechanism

\begin{abstract}
A B S T R A C T
Palladium-catalyzed asymmetric allylic alkylation (AAA) of vinyl benzoxazinanones has become an important strategy for the synthesis of chiral nitrogen-containing heterocycle compounds. However, the asymmetric synthesis of linear-selective products has rarely been reported. The simultaneous control of regio-, $E / Z$ - and enantioselectivities constitutes a major challenge and inhibits the advancement of this chemistry. Herein, we present a palladium-catalyzed AAA of vinyl benzoxazinanones with $\alpha$-thiocyanato ketones, affording various chiral thiocyanates characterized with high linear-, $E$ - and stereoselectivities. The reaction has a broad substrate scope and the chiral thiocyanates can be transformed to useful heterocycles. Experimental and computational studies suggest an inner-sphere mechanism for AAA process, which results from the acidic and coordination effect of the nucleophilic substrates with palladium catalyst.
\end{abstract}

(C) 2021, Dalian Institute of Chemical Physics, Chinese Academy of Sciences. Published by Elsevier B.V. All rights reserved.

\section{Introduction}

Vinyl benzoxazinanones as substrates have recently received enormous attention in synthetic chemistry $[1,2]$ because of its huge potential in the construction of nitrogenous skeletons which widely exist in natural alkaloids and bioactive compounds [3-6]. In the presence of chiral $\mathrm{Pd}(0)$ catalysts, vinyl benzoxazinanones can easily generate Pd-stabilized zwitterionic intermediates which could act as 1,4-carbon-nitrogen dipole to react with a wide range of nucleophiles [7-9] or sulfur ylides [10-12], electron-deficient alkenes [13-20] and enals $[21,22]$ respectively, enabling the construction of chiral arylamine or five-, six-, seven-membered nitrogen-containing het- erocycle compounds. Nevertheless, despite these notable advancements in the chemistry of vinyl benzoxazinanones, there still remains a major challenge to be addressed that most of reactions afford branch-selective products via zwitterionic $\pi$-allyl palladium intermediates. To date, there exist only two examples on the terminal substituted allylation reaction of vinyl benzoxazinanones in racemic version (Scheme 1(b)) $[23,24]$. To the best of our knowledge, the Pd(0)-catalyzed linear selective asymmetric allylic alkylation (AAA) of vinyl benzoxazinanones has never been realized. The development of this linear selective AAA may not only improve the chemistry of vinyl benzoxazinanones, but also create new reactions for the synthesis of useful chiral arylamines [25-30]. In the traditional

\footnotetext{
*Corresponding author. Tel: +86-411-84379913; Fax: +86-411-84675584; E-mail: fanhj@dicp.ac.cn

\# Corresponding author. Tel: +86-411-84379252; Fax: +86-411-84694447; E-mail: yanliu503@dicp.ac.cn

\$Corresponding author. Tel: +86-411-84379070; Fax: +86-411-84694447; E-mail: canli@dicp.ac.cn

This work was supported by the National Natural Science Foundation of China $(21673224,21871254,21532006,21472187)$, Dalian Institute of Chemical Physics (DICP ZZBS201602) and the Strategic Priority Research Program of the Chinese Academy of Sciences (XDB17010200).

DOI: 10.1016/S1872-2067(20)63751-2 | http://www.sciencedirect.com/journal/chinese-journal-of-catalysis | Chin. J. Catal., Vol. 42, No. 7, July 2021
} 
a) Pd-catalyzed decarboxylative reaction of vinyl benzoxazinanones
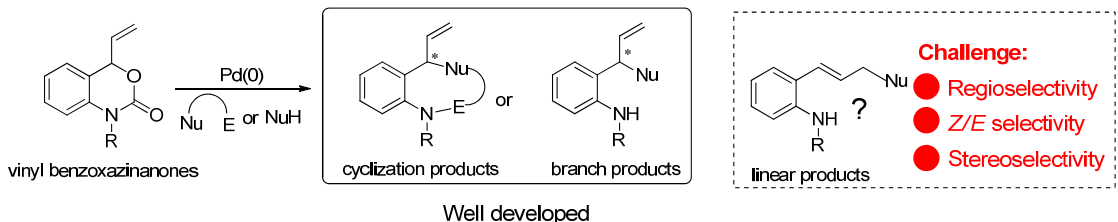

Well developed

b) Rh or Ir/Brnøsted acid catalyzed linear allylic substitution (Racemic only)

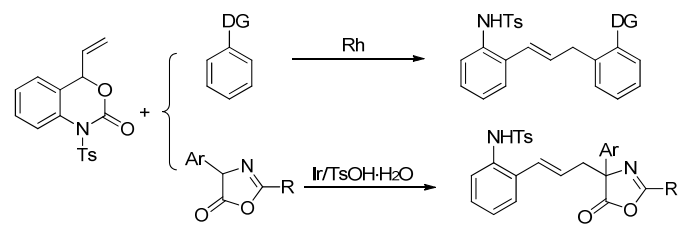

c) The reaction model of $\mathrm{Pd}(0)$-catalyzed allylic substitution of vinyl benzoxazinanones

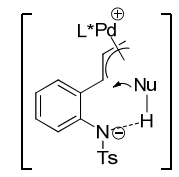

Out-sphere mechanism branch products

VS

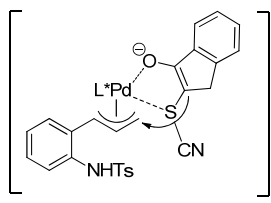

Inner-sphere mechanism linear products (this work)

d) This work: $\operatorname{Pd}(0)$-catalyzed asymmetric terminal allylic substitution with $\alpha$-thiocyanate ketones.

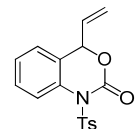

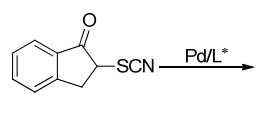<smiles>CCCCCC1(CC=Nc2ccccc2C#N)Cc2ccccc2C1=O</smiles>

up to $95 \%$ ee

- linear/branch >20/1

- $E / Z>20 / 1$

Scheme 1. The chemistry of vinyl benzoxazinanones.

$\operatorname{Pd}(0)$-catalyzed AAA reactions, the kinetically controlled linear products were achieved due to the steric hindrance effect [31-37]. However, nucleophilic attack at the more hindered allyl sites occurred in the AAA of vinyl benzoxazinanones presumably because of the direction effect of tosylamide through hydrogen-bonding [8,9] or ion pair interaction [10] with nucleophiles (Scheme 1(c)). Thus, the key to achieve linear selective AAA lies in the destruction of this kind of six-membered transition state.

Thiocyanates are a kind of important compounds found in naturally occurring marine sponges and alkaloids, some of which are reported to display good anti-fungal and anti-fouling activity [38-43]. However, the efficient method to prepare chiral compounds bearing thiocyanato-attached stereocenters remains scarce [44-46]. In our recent work, $\alpha$-thiocyanato ketones were proven to be efficient nucleophiles in the asymmetric reaction with $o-\mathrm{QM}$ [47]. The importance of chiral thiocyanates coupled with our ongoing interest in developing new asymmetic reactions prompted our investigation into the AAA of $\alpha$-thiocyanato ketones with vinyl benzoxazinanones. However, the simultaneous control of regioselectivity, $E / Z$ selectivity and stereoselectivity constitutes a great challenge of our designed reaction.

Herein, we report a $\mathrm{Pd}(0)$-catalyzed linear selective AAA with vinyl benzoxazinanones. In the presence of chiral BINOL-based phosphoramidite, the AAA of $\alpha$-thiocyanato ketones to vinyl benzoxazinanones produced chiral thiocyanates with quaternary stereo centers in high enantioselectivities (up to $95 \%$ ee) (Scheme 1(d)). The reaction has a broad substrate scope and the chiral thiocyanates can be transformed to chiral thiazolidine and tetrazole compounds. Mechanistic experiments suggested that the destruction of hydrogen-bond directing effect by the deprotonation of $\alpha$-thiocyanato ketones may play an important role on the control of linear selectivity.

\section{Experimental}

\subsection{General information}

Unless otherwise noted, materials were purchased from commercial suppliers and used without further purification. All the dry solvents were treated according to standard methods or purchased from J\&K. TLC was performed with silica gel GF254 precoated on glass plates and spots were visualized with UV. Flash column chromatography was performed using 300-400 mesh silica gel. NMR spectra were recorded with a Bruker Avance II 400 NMR spectrometer. Chemical shifts $(\delta)$ are reported in ppm from the resonance of tetramethyl silane as the internal standard (TMS: $0.00 \mathrm{ppm}$ ). Data are reported as follows: chemical shift, multiplicity $(\mathrm{s}=$ singlet, $\mathrm{d}=$ doublet, $\mathrm{t}=$ triplet, $\mathrm{q}=$ quartert, $\mathrm{m}=$ multiplet, $\mathrm{br}=$ broad, $\mathrm{dd}=$ doublet of doublets), coupling constants (Hz) and integration. HPLC analysis was performed on Agilent HPLC 1100 or 1260 equipped with Daicel chiral column. High resolution mass spectra for all the unknown compounds were done by an LTQ-Orbitrap instrument (ESI) (Thermo Fisher Scientific, USA). All air- and 
moisture-sensitive reactions were performed under an atmosphere of Ar in fire dried Schlenk tube.

\subsection{General procedure for the synthesis of product 3}

To a flame dried Schlenk tube under Ar atmosphere was added $\mathrm{Pd}_{2}(\mathrm{dba})_{3} \cdot \mathrm{CHCl}_{3}(5 \mathrm{~mol} \%)$ and chiral ligand $\mathbf{L 1}$ (11 mol\%). $2.0 \mathrm{~mL}$ DCE was added sequentially and the mixture was stirred at room temperature for $10 \mathrm{~min}$. After that, the glass tube was cooled to $0{ }^{\circ} \mathrm{C}$ and stirred for $10 \mathrm{~min}$. Finally, the substrate 1 ( $0.2 \mathrm{mmol}), 2(0.4 \mathrm{mmol})$ and $\mathrm{K}_{2} \mathrm{CO}_{3}$ (2.0 equiv.) were added to the mixture and reacted for $24 \mathrm{~h}$. After the reaction was complete, saturated $\mathrm{NH}_{4} \mathrm{Cl}$ solution $(5 \mathrm{~mL})$ was added to quench the reaction. The organic phase was separated, and the aqueous layer was washed with dichloromethane $(5 \mathrm{~mL} \times$ 2). The combined organic layer was dried by $\mathrm{Na}_{2} \mathrm{SO}_{4}$, filtered, concentrated under reduced pressure. The crude product was purified by column chromatography to afford the target product 3 (petrol ether $/$ EtOAc = 3/1).

\subsection{General procedure for the synthesis of product 9}

To a flame dried Schlenk tube under Ar atmosphere was added $\mathrm{Pd}_{2}(\mathrm{dba})_{3} \cdot \mathrm{CHCl}_{3}(5 \mathrm{~mol} \%)$ and chiral ligand $\mathbf{L 1}$ (11 mol\%). DCE (0.1 M) was added sequentially and the mixture was stirred at room temperature for $10 \mathrm{~min}$. After that, the glass tube was cooled to $0{ }^{\circ} \mathrm{C}$ and stirred for $10 \mathrm{~min}$. Finally, the substrate 1 ( $0.1 \mathrm{mmol}$ or $0.05 \mathrm{mmol}$ ), 8 (1.5 eq) and $\mathrm{K}_{2} \mathrm{CO}_{3}$ (2.0 equiv.) were added to the mixture and reacted for 2-4 h. After the reaction was complete, saturated $\mathrm{NH}_{4} \mathrm{Cl}$ solution $(5 \mathrm{~mL}$ ) was added to quench the reaction. The organic phase was separated, and the aqueous layer was washed with dichloromethane $(5 \mathrm{~mL} \times 2)$. The combined organic layer was dried by $\mathrm{Na}_{2} \mathrm{SO}_{4}$, filtered, concentrated under reduced pressure. The crude product was purified by column chromatography to afford the target product 9 (petrol ether $/$ EtOAc $=3 / 1$ ).

\subsection{Spectral data for products}

3aa. Yellow solid, $63.2 \mathrm{mg}$, $67 \%$ yield, $91 \%$ ee. $[\alpha]_{\mathrm{D}} \mathrm{D}^{20}=-59.5$ (c = 0.63, $\left.\mathrm{CH}_{3} \mathrm{CN}\right) .{ }^{1} \mathrm{H}$ NMR $\left(400 \mathrm{MHz}, \mathrm{CDCl}_{3}\right): \delta(\mathrm{ppm}) 7.85$ (d, $J$ $=7.7 \mathrm{~Hz}, 1 \mathrm{H}), 7.71-7.64(\mathrm{~m}, 1 \mathrm{H}), 7.61(\mathrm{~d}, J=8.3 \mathrm{~Hz}, 2 \mathrm{H}), 7.44$ (m, 2H), 7.25-7.06 (m, 6H), $6.92(\mathrm{br}, 1 \mathrm{H}), 6.54(\mathrm{~d}, J=15.5 \mathrm{~Hz}$, 1H), 5.95-5.81 (m, 1H), 3.58 (d, $J=18.3 \mathrm{~Hz}, 1 \mathrm{H}), 3.29$ (d, $J=$ $18.3 \mathrm{~Hz}, 1 \mathrm{H}), 2.93-2.80(\mathrm{~m}, 2 \mathrm{H}), 2.40(\mathrm{~s}, 3 \mathrm{H}) .{ }^{13} \mathrm{C}$ NMR $(100$ $\left.\mathrm{MHz}, \mathrm{CDCl}_{3}\right): \delta$ (ppm) 199.59, 149.78, 144.03, 136.58, 136.44, 133.36, 133.14, 132.31, 131.04, 129.72, 128.78, 128.68, 127.28, 127.09, 126.79, 126.60, 125.67, 125.61, 125.49, 109.83, 59.26, 40.13, 38.78, 21.60. HRMS (ESI): calculated for $\mathrm{C}_{26} \mathrm{H}_{22} \mathrm{~N}_{2} \mathrm{O}_{3} \mathrm{~S}_{2}$ $[\mathrm{M}+\mathrm{H}]^{+}:$475.1145, found: 475.1140. HPLC: Chiralpak AD-H column, hexane $/ i-\mathrm{PrOH}=80 / 20$, flow $=1 \mathrm{~mL} / \mathrm{min}, 235 \mathrm{~nm}, 25$ ${ }^{\circ} \mathrm{C}$, retention time $34.347 \mathrm{~min}$ (major) and $37.342 \mathrm{~min}$.

3ab. Yellow solid. $49.1 \mathrm{mg}, 44 \%$ yield, $90 \%$ ee. $[\alpha]_{\mathrm{D}^{20}=-27.4}$ (c = 0.49, $\left.\mathrm{CH}_{3} \mathrm{CN}\right) .{ }^{1} \mathrm{H}$ NMR (400MHz, DMSO-d $): \delta(\mathrm{ppm}) 9.70$ (s, $1 \mathrm{H}), 8.05(\mathrm{~d}, J=7.6 \mathrm{~Hz}, 1 \mathrm{H}), 7.86(\mathrm{~d}, J=7.5 \mathrm{~Hz}, 1 \mathrm{H})$, 7.57-7.51 (m, 3H), $7.47(\mathrm{~m}, 1 \mathrm{H}), 7.33$ (d, $J=8.1 \mathrm{~Hz}, 2 \mathrm{H}), 7.16$ (m, 2H), $6.97(\mathrm{~m}, 1 \mathrm{H}), 6.77(\mathrm{~d}, J=15.7 \mathrm{~Hz}, 1 \mathrm{H}), 6.17-5.99(\mathrm{~m}$,
1H), 3.62 (d, $J=18.8 \mathrm{~Hz}, 1 \mathrm{H}), 3.27$ (d, $J=18.8 \mathrm{~Hz}, 1 \mathrm{H}), 2.84$ (ddd, $J=22.4,14.5,7.1 \mathrm{~Hz}, 2 \mathrm{H}), 2.35$ (s, 3H). ${ }^{13} \mathrm{C}$ NMR $(100 \mathrm{MHz}$, DMSO-d6): $\delta$ (ppm) 199.44, 150.10, 143.52, 139.57, 137.66, 135.60, 133.97, 133.58, 131.37, 131.13, 130.03, 128.65, 127.61, 127.22 (two C signals overlapped), 126.64, 124.54, 124.15, $121.65,110.40,59.34,39.13,38.62,21.46$. HRMS (ESI): calculated for $\mathrm{C}_{26} \mathrm{H}_{21} \mathrm{BrN}_{2} \mathrm{O}_{3} \mathrm{~S}_{2} \quad\left[\mathrm{M}+\mathrm{NH}_{4}\right]^{+}:$570.0515, found: 570.0519. HPLC: Chiralpak AD-H column, hexane $/ i-\mathrm{PrOH}=$ $60 / 40$, flow $=0.7 \mathrm{~mL} / \mathrm{min}, 235 \mathrm{~nm}, 25{ }^{\circ} \mathrm{C}$, retention time $19.629 \mathrm{~min}$ (major) and $29.208 \mathrm{~min}$.

3ac. Yellow solid, $60.5 \mathrm{mg}, 62 \%$ yield, $90 \%$ ee. $[\alpha]_{\mathrm{D}}{ }^{20}=-56.6$ (c = 0.61, $\left.\mathrm{CH}_{3} \mathrm{CN}\right) .{ }^{1} \mathrm{H}$ NMR $\left(400 \mathrm{MHz}, \mathrm{CDCl}_{3}\right): \delta(\mathrm{ppm}) 7.68(\mathrm{~d}, J$ $=7.6 \mathrm{~Hz}, 1 \mathrm{H}), 7.60(\mathrm{~d}, J=8.3 \mathrm{~Hz}, 2 \mathrm{H}), 7.47(\mathrm{~d}, J=7.3 \mathrm{~Hz}, 1 \mathrm{H})$, $7.37(\mathrm{t}, J=7.5 \mathrm{~Hz}, 1 \mathrm{H}), 7.22(\mathrm{~d}, J=8.4 \mathrm{~Hz}, 3 \mathrm{H}), 7.16-7.07(\mathrm{~m}$, $3 \mathrm{H}), 7.00(\mathrm{~s}, 1 \mathrm{H}), 6.60(\mathrm{~d}, J=15.6 \mathrm{~Hz}, 1 \mathrm{H}), 5.96-5.82(\mathrm{~m}, 1 \mathrm{H})$, 3.47 (d, $J=18.3 \mathrm{~Hz}, 1 \mathrm{H}$ ), 3.16 (d, $J=18.3 \mathrm{~Hz}, 1 \mathrm{H}), 2.96-2.79$ (m, 2H), 2.39 (s, 3H), 2.29 (s, 3H). ${ }^{13} \mathrm{C}$ NMR (100 MHz, $\left.\mathrm{CDCl}_{3}\right): \delta$ (ppm) 199.99, 148.81, 144.02, 137.09, 136.40, 136.10, 133.15 (two C signals overlapped), 132.54, 131.09, 129.71, 128.92, 128.62, 127.30, 127.03, 126.84, 125.74, 125.49, 122.81, 109.97, 59.23, 40.18, 37.69, 21.60, 17.77. HRMS (ESI): calculated for $\mathrm{C}_{27} \mathrm{H}_{24} \mathrm{~N}_{2} \mathrm{O}_{3} \mathrm{~S}_{2}[\mathrm{M}+\mathrm{H}]^{+}:$489.1301, found: 489.1310. HPLC: Chiralpak AD-H column, hexane $/ i-\mathrm{PrOH}=80 / 20$, flow $=1.0$ $\mathrm{mL} / \mathrm{min}, 235 \mathrm{~nm}, 25^{\circ} \mathrm{C}$, retention time $26.148 \mathrm{~min}$ (major) and 35.327 min.

3ad. Yellow solid, $58.9 \mathrm{mg}$, $53 \%$ yield, $90 \%$ ee. $[\alpha]_{\mathrm{D}}{ }^{20}=-14.5$ (c = 0.29, $\left.\mathrm{CH}_{3} \mathrm{CN}\right) .{ }^{1} \mathrm{H}$ NMR $\left(400 \mathrm{MHz}, \mathrm{CDCl}_{3}\right): \delta(\mathrm{ppm}) 7.69(\mathrm{~d}, J$ $=9.0 \mathrm{~Hz}, 2 \mathrm{H}), 7.60(\mathrm{~m}, 4 \mathrm{H}), 7.24(\mathrm{~d}, J=8.0 \mathrm{~Hz}, 2 \mathrm{H}), 7.14(\mathrm{~m}, 3 \mathrm{H})$, $6.96(\mathrm{~s}, 1 \mathrm{H}), 5.94-5.80(\mathrm{~m}, 1 \mathrm{H}), 3.61(\mathrm{~d}, J=18.5 \mathrm{~Hz}, 1 \mathrm{H}), 3.24(\mathrm{~d}$, $J=18.5 \mathrm{~Hz}, 1 \mathrm{H}), 2.88(\mathrm{~d}, J=7.2 \mathrm{~Hz}, 2 \mathrm{H}), 2.41(\mathrm{~s}, 3 \mathrm{H}) .{ }^{13} \mathrm{C} \mathrm{NMR}$ (100 MHz, $\left.\mathrm{CDCl}_{3}\right): \delta$ (ppm) 198.49, 151.32, 144.10, 136.31, $133.10,132.45,132.34,132.22,132.17,131.35,129.93,129.76$, 128.75, 127.29, 127.04, 126.90, 126.55, 125.72, 125.10, 109.49, 59.08, 39.97, 38.23, 21.62. HRMS (ESI): calculated for $\mathrm{C}_{26} \mathrm{H}_{22} \mathrm{BrN}_{2} \mathrm{O}_{3} \mathrm{~S}_{2}[\mathrm{M}+\mathrm{H}]^{+}$: 553.0250, found: 553.0247. HPLC: Chiralpak AD-H column, hexane $/ i-\mathrm{PrOH}=80 / 20$, flow $=1.0$ $\mathrm{mL} / \mathrm{min}, 235 \mathrm{~nm}, 25^{\circ} \mathrm{C}$, retention time $37.536 \mathrm{~min}$ (major) and $43.268 \mathrm{~min}$.

3ae. Yellow solid, $38.1 \mathrm{mg}, 37 \%$ yield, $92 \%$ ee. $[\alpha]_{\mathrm{D}}{ }^{20}=-21.3$ (c $\left.=0.38, \mathrm{CH}_{3} \mathrm{CN}\right) .{ }^{1} \mathrm{H}$ NMR $(400 \mathrm{MHz}$, DMSO-d 6$): \delta(\mathrm{ppm}) 9.69$ (s, 1H), $7.83(\mathrm{~d}, J=8.2 \mathrm{~Hz}, 1 \mathrm{H}), 7.72(\mathrm{~d}, J=1.1 \mathrm{~Hz}, 1 \mathrm{H}), 7.60$ (dd, $J=8.3,1.7 \mathrm{~Hz}, 1 \mathrm{H}), 7.53(\mathrm{~d}, J=8.2 \mathrm{~Hz}, 2 \mathrm{H}), 7.40(\mathrm{~m}, 1 \mathrm{H}), 7.33(\mathrm{~d}$, $J=8.1 \mathrm{~Hz}, 2 \mathrm{H}), 7.21-7.11(\mathrm{~m}, 2 \mathrm{H}), 6.96(\mathrm{~m}, 1 \mathrm{H}), 6.73(\mathrm{~d}, J=15.7$ $\mathrm{Hz}, 1 \mathrm{H}), 6.05-5.93(\mathrm{~m}, 1 \mathrm{H}), 3.69(\mathrm{~d}, J=18.8 \mathrm{~Hz}, 1 \mathrm{H}), 3.36(\mathrm{~d}, J=$ $18.8 \mathrm{~Hz}, 1 \mathrm{H}), 2.81(\mathrm{~m}, 2 \mathrm{H}), 2.35$ (s, 3H). ${ }^{13} \mathrm{C}$ NMR (100 MHz, DMSO-d 6 ): $\delta$ (ppm) 198.91, 152.65, 143.52, 141.91, 137.66, 133.97, 133.56, 132.15, 131.16, 130.02, 129.65, 128.66, 127.65, 127.42, 127.21 (two C signals overlapped), 126.84, 126.51, 124.07, 110.46, 59.55, 38.89, 37.80, 21.45. HRMS (ESI): calculated for $\mathrm{C}_{26} \mathrm{H}_{25} \mathrm{ClN}_{3} \mathrm{O}_{3} \mathrm{~S}_{2}\left[\mathrm{M}+\mathrm{NH}_{4}\right]+$ : 526.1020 , found: 526.1020 . HPLC: Chiralpak IA column, hexane $/ i-\mathrm{PrOH}=80 / 20$, flow $=1.0$ $\mathrm{mL} / \mathrm{min}, 235 \mathrm{~nm}, 25^{\circ} \mathrm{C}$, retention time $23.473 \mathrm{~min}$ (major) and $26.841 \mathrm{~min}$.

3af. Yellow solid, $32.5 \mathrm{mg}$, 33\% yield, $90 \%$ ee. $[\alpha]_{\mathrm{D}}{ }^{20}=-13.6$ (c = 0.33, $\left.\mathrm{CH}_{3} \mathrm{CN}\right) .{ }^{1} \mathrm{H}$ NMR (400MHz, DMSO-d 6$): \delta(\mathrm{ppm}) 9.68$ (s, $1 \mathrm{H}), 7.70(\mathrm{~d}, J=7.9 \mathrm{~Hz}, 1 \mathrm{H}), 7.52(\mathrm{~d}, J=8.2 \mathrm{~Hz}, 2 \mathrm{H})$, 7.41-7.30 (m, 5H), 7.19-7.09 (m, 2H), 6.99-6.92 (m, 1H), 6.71 
(d, $J=15.7 \mathrm{~Hz}, 1 \mathrm{H}), 6.03-5.91(\mathrm{~m}, 1 \mathrm{H}), 3.62(\mathrm{~d}, J=18.6 \mathrm{~Hz}, 1 \mathrm{H})$, 3.28 (d, $J=18.7 \mathrm{~Hz}, 1 \mathrm{H}), 2.84-2.69(\mathrm{~m}, 2 \mathrm{H}), 2.43(\mathrm{~s}, 3 \mathrm{H}), 2.34(\mathrm{~s}$, 3H). ${ }^{13} \mathrm{C}$ NMR (100 MHz, DMSO-d6): $\delta$ (ppm) 199.46, 151.17, 148.33, 143.51, 137.68, 133.95, 133.53, 131.01, 130.96, 130.29, $130.03,128.63,127.65,127.45,127.20$ (two C signals overlapped), 126.48, 125.07, 124.24, 110.65, 59.57, 39.21, 37.86, 22.19, 21.45. HRMS (ESI): calculated for $\mathrm{C}_{27} \mathrm{H}_{25} \mathrm{~N}_{2} \mathrm{O}_{3} \mathrm{~S}_{2}[\mathrm{M}+\mathrm{H}]^{+}$: 489.1301, found: 489.1305. HPLC: Chiralpak AD-H column, hexane $/ i-\mathrm{PrOH}=85 / 15$, flow $=1.0 \mathrm{~mL} / \mathrm{min}, 235 \mathrm{~nm}, 25{ }^{\circ} \mathrm{C}$, retention time $56.318 \mathrm{~min}$ (major) and $59.608 \mathrm{~min}$.

3ag. Yellow solid, $51.9 \mathrm{mg}, 51 \%$ yield, $95 \%$ ee. $[\alpha]_{\mathrm{D}^{20}=-41.3}$ (c $=0.52, \mathrm{CH}_{3} \mathrm{CN}$ ). ${ }^{1} \mathrm{H}$ NMR $\left(400 \mathrm{MHz}, \mathrm{CDCl}_{3}\right): \delta(\mathrm{ppm}) 7.78(\mathrm{~d}, \mathrm{~J}$ $=8.6 \mathrm{~Hz}, 1 \mathrm{H}), 7.61(\mathrm{~d}, J=8.3 \mathrm{~Hz}, 2 \mathrm{H}), 7.23(\mathrm{~d}, J=8.1 \mathrm{~Hz}, 2 \mathrm{H})$, $7.20(\mathrm{~d}, J=7.3 \mathrm{~Hz}, 1 \mathrm{H}), 7.18-7.14(\mathrm{~m}, 2 \mathrm{H}), 7.14-7.07(\mathrm{~m}, 1 \mathrm{H})$, $6.97(\mathrm{dd}, J=8.6,2.0 \mathrm{~Hz}, 1 \mathrm{H}), 6.86(\mathrm{~s}, 1 \mathrm{H}), 6.77(\mathrm{~s}, 1 \mathrm{H}), 6.49$ (d, $J$ $=15.6 \mathrm{~Hz}, 1 \mathrm{H}), 5.94-5.82(\mathrm{~m}, 1 \mathrm{H}), 3.87(\mathrm{~s}, 3 \mathrm{H}), 3.53(\mathrm{~d}, J=18.3$ $\mathrm{Hz}, 1 \mathrm{H}), 3.30$ (d, $J=18.3 \mathrm{~Hz}, 1 \mathrm{H}), 2.40(\mathrm{~s}, 3 \mathrm{H}) .{ }^{13} \mathrm{C}$ NMR $(100$ $\left.\mathrm{MHz}, \mathrm{CDCl}_{3}\right): \delta$ (ppm) 197.61, 166.86, 153.07, 144.02, 136.46, 133.13, 132.32, 130.91, 129.71, 128.64, 127.27, 127.23, 127.17, 126.75, 126.37, 125.94, 125.56, 117.04, 110.13, 109.61, 59.95, 55.91, 40.52, 39.05, 21.59. HRMS (ESI): calculated for $\mathrm{C}_{27} \mathrm{H}_{25} \mathrm{~N}_{2} \mathrm{O}_{4} \mathrm{~S}_{2}[\mathrm{M}+\mathrm{H}]+:$ 505.1250, found: 505.1254. HPLC: Chiralpak IA column, hexane $/ i-\mathrm{PrOH}=85 / 15$, flow $=1.0 \mathrm{~mL} / \mathrm{min}$, $235 \mathrm{~nm}, 25^{\circ} \mathrm{C}$, retention time $50.364 \mathrm{~min}$ (major) and 56.340 min.

3ah. Yellow solid, $63.1 \mathrm{mg}, 64 \%$ yield, $90 \%$ ee. $[\alpha]_{\mathrm{D}^{20}=-38.7}$ (c = 0.63, $\mathrm{CH}_{3} \mathrm{CN}$ ). ${ }^{1} \mathrm{H}$ NMR (400MHz, DMSO-d6): $\delta$ (ppm) 9.70 (s, 1H), 7.69-7.65 (m, 2H), 7.64-7.60 (m, 1H), $7.54(\mathrm{~d}, J=8.2 \mathrm{~Hz}$, $2 \mathrm{H}), 7.45-7.39(\mathrm{~m}, 1 \mathrm{H}), 7.33(\mathrm{~d}, J=8.1 \mathrm{~Hz}, 2 \mathrm{H}), 7.20-7.11(\mathrm{~m}$, 2H), 7.01-6.94 (m, 1H), $6.75(\mathrm{~d}, J=15.7 \mathrm{~Hz}, 1 \mathrm{H}) 6.08-5.96(\mathrm{~m}$, 1H), 3.66 (d, $J=18.3 \mathrm{~Hz}, 1 \mathrm{H}), 3.32$ (d, $J=18.4 \mathrm{~Hz}, 1 \mathrm{H}), 2.90-2.73$ (m, 2H), 2.35 (s, 3H). ${ }^{13} \mathrm{C}$ NMR (100 MHz, DMSO-d 6 ): $\delta$ (ppm) 199.40 (d, $J=3.0 \mathrm{~Hz}$ ), 162.58 (d, $J=246.9 \mathrm{~Hz}$ ), 146.70, 146.68, 143.51, 137.68, 135.13 (d, $J=7.8 \mathrm{~Hz}$ ), 133.97, 133.57, 131.15, 130.02, 129.41 (d, $J=8.3 \mathrm{~Hz}$ ), 128.66, 127.67, 127.22(two C signals overlapped), 126.51, $124.61(\mathrm{~d}, J=23.6 \mathrm{~Hz}), 124.10$, 110.91 (d, $J=22.3 \mathrm{~Hz}$ ), 110.45, 60.09, 38.85, 37.47, 21.45. ${ }^{19} \mathrm{~F}$ NMR (376 MHz, DMSO-d 6 ): $\delta-112.95$. HRMS (ESI): Calculated for $\mathrm{C}_{26} \mathrm{H}_{21} \mathrm{FN}_{2} \mathrm{O}_{3} \mathrm{~S}_{2}\left[\mathrm{M}+\mathrm{NH}_{4}\right]^{+}$: 510.1316, found: 510.1321 . HPLC: Chiralpak IC column, hexane $/ i-\mathrm{PrOH}=60 / 40$, flow $=1.0$ $\mathrm{mL} / \mathrm{min}, 235 \mathrm{~nm}, 25^{\circ} \mathrm{C}$, retention time $44.564 \mathrm{~min}$ (major) and 39.402 min.

3ai. Yellow solid, $53.7 \mathrm{mg}, 49 \%$ yield, $91 \%$ ee. $[\alpha]_{\mathrm{D}}{ }^{20}=-89.4$ (c $=0.54, \mathrm{CH}_{3} \mathrm{CN}$ ). ${ }^{1} \mathrm{H}$ NMR (400 MHz, DMSO-d $): \delta 9.70(\mathrm{~s}, 1 \mathrm{H}$ ), 8.00-7.93 (m, 2H), 7.57 (d, $J=8.0 \mathrm{~Hz}, 1 \mathrm{H}), 7.53(\mathrm{~d}, J=8.2 \mathrm{~Hz}$, $2 \mathrm{H}), 7.44-7.39(\mathrm{~m}, 1 \mathrm{H}), 7.33(\mathrm{~d}, J=8.1 \mathrm{~Hz}, 2 \mathrm{H}), 7.20-7.11(\mathrm{~m}$, 2H), 7.01-6.92 (m, 1H), $6.74(\mathrm{~d}, \mathrm{~J}=15.7 \mathrm{~Hz}, 1 \mathrm{H}), 6.10-5.94(\mathrm{~m}$, $1 \mathrm{H}), 3.64(\mathrm{~d}, J=18.8 \mathrm{~Hz}, 1 \mathrm{H}), 3.31(\mathrm{~d}, J=18.8 \mathrm{~Hz}, 1 \mathrm{H}), 2.81$ (ddd, $J=22.1,14.5,7.2 \mathrm{~Hz}, 2 \mathrm{H}), 2.35$ (s, 3H). ${ }^{13} \mathrm{C}$ NMR (101 MHz, DMSO-d 6 ): $\delta$ 198.90, 149.78, 143.51, 139.40, 137.66, 135.38, 133.96, 133.55, 131.17, 130.02, 129.53, 128.67, 127.65, 127.58, 127.22(two C signals overlapped), 126.52, 124.07, 122.19, 110.42, 59.67, 38.78, 37.66, 21.47. HRMS (ESI): calculated for $\mathrm{C}_{26} \mathrm{H}_{21} \mathrm{BrN}_{2} \mathrm{O}_{3} \mathrm{~S}_{2}\left[\mathrm{M}+\mathrm{NH}_{4}\right]^{+}: 570.0515$, found: 570.0517. HPLC: Chiralpak OD-H column, hexane $/ \mathrm{i}-\mathrm{PrOH}=80 / 20$, flow $=1.0$ $\mathrm{mL} / \mathrm{min}, 235 \mathrm{~nm}, 25^{\circ} \mathrm{C}$, retention time $35.559 \mathrm{~min}$ (major) and $30.662 \mathrm{~min}$ 3aj. Yellow solid, $57.4 \mathrm{mg}, 59 \%$ yield, $93 \%$ ee. $[\alpha]_{\mathrm{D}^{20}}=-75.6$ (c $=0.57, \mathrm{CH}_{3} \mathrm{CN}$ ). ${ }^{1} \mathrm{H}$ NMR (400 MHz, DMSO-d $): \delta 9.70(\mathrm{~s}, 1 \mathrm{H})$, 7.62 (d, $J=4.6 \mathrm{~Hz}, 2 \mathrm{H}), 7.53(\mathrm{~d}, J=8.3 \mathrm{~Hz}, 2 \mathrm{H}), 7.49$ (d, $J=8.3$ $\mathrm{Hz}, 1 \mathrm{H}$ ), 7.38 (dd, $J=7.3,2.1 \mathrm{~Hz}, 1 \mathrm{H}), 7.33$ (d, $J=8.0 \mathrm{~Hz}, 2 \mathrm{H}$ ), $7.20-7.10(\mathrm{~m}, 2 \mathrm{H}), 6.98(\mathrm{dt}, J=7.1,3.6 \mathrm{~Hz}, 1 \mathrm{H}), 6.72(\mathrm{~d}, J=15.7$ $\mathrm{Hz}, 1 \mathrm{H}), 6.05-5.92$ (m, 1H), 3.62 (d, $J=18.5 \mathrm{~Hz}, 1 \mathrm{H}), 3.27$ (d, $J=$ $18.5 \mathrm{~Hz}, 1 \mathrm{H}), 2.89-2.69$ (m, 2H), 2.40 (s, 3H), 2.35 (s, 3H). ${ }^{13} \mathrm{C}$ NMR (101 MHz, DMSO-d $)$ ): $\delta$ 200.06, 148.09, 143.51, 138.91, $138.18,137.68,133.95,133.53,133.42,131.01,130.03,128.64$, 127.65, 127.21(two C signals overlapped), 127.03, 126.48, $124.91,124.24,110.62,59.64,39.11,37.66,21.45,21.04$. HRMS (ESI): calculated for $\mathrm{C}_{27} \mathrm{H}_{24} \mathrm{~N}_{2} \mathrm{O}_{3} \mathrm{~S}_{2}[\mathrm{M}+\mathrm{H}]^{+}$: 489.1301, found: 489.1307. HPLC: Chiralpak AD-H column, hexane $/ i-\mathrm{PrOH}=$ $80 / 20$, flow $=1.0 \mathrm{~mL} / \mathrm{min}, 235 \mathrm{~nm}, 25{ }^{\circ} \mathrm{C}$, retention time $36.223 \mathrm{~min}$ (major) and $42.376 \mathrm{~min}$.

3ak. Yellow solid, $72.0 \mathrm{mg}, 71 \%$ yield, $90 \%$ ee. $[\alpha]_{\mathrm{D}}{ }^{20}=-85.6$ (c = 0.72, $\mathrm{CH}_{3} \mathrm{CN}$ ). ${ }^{1} \mathrm{H}$ NMR (400 MHz, $\mathrm{CDCl}_{3}$ ): $\delta 7.61(\mathrm{~d}, J=8.3$ $\mathrm{Hz}, 2 \mathrm{H}), 7.32$ (d, $J=8.1 \mathrm{~Hz}, 1 \mathrm{H}), 7.26(\mathrm{~s}, 2 \mathrm{H}), 7.23(\mathrm{~d}, J=8.1 \mathrm{~Hz}$, 2H), 7.21-7.19 (m, 1H), 7.18-7.15 (m, 2H), 7.14-7.08 (m, 1H), 6.81 (brs, 1H), 6.51 (d, $J=15.6 \mathrm{~Hz}, 1 \mathrm{H}), 5.94-5.80(\mathrm{~m}, 1 \mathrm{H}), 3.86$ (d, $J=6.0 \mathrm{~Hz}, 3 \mathrm{H}$ ), 3.49 (d, $J=18.0 \mathrm{~Hz}, 1 \mathrm{H}), 3.21$ (d, $J=18.0 \mathrm{~Hz}$, $1 \mathrm{H}), 2.86(\mathrm{~d}, J=7.3 \mathrm{~Hz}, 2 \mathrm{H}), 2.40(\mathrm{~s}, 3 \mathrm{H}) .{ }^{13} \mathrm{C}$ NMR (101 MHz, $\left.\mathrm{CDCl}_{3}\right): \delta 199.59,160.30,144.02,142.57,136.45,134.58$, 133.15, 132.24, 131.04, 129.71, 128.68, 127.31, 127.29, 127.14, 126.74, 126.15, 125.78, 125.53, 109.86, 106.28, 59.94, 55.75, $40.17,38.25,21.60$. HRMS (ESI): calculated for $\mathrm{C}_{27} \mathrm{H}_{24} \mathrm{~N}_{2} \mathrm{O}_{4} \mathrm{~S}_{2}$ $[\mathrm{M}+\mathrm{H}]^{+}$: 505.1250, found: 505.1260. HPLC: Chiralpak AD-H column, hexane $/ \mathrm{i}-\mathrm{PrOH}=80 / 20$, flow $=1.0 \mathrm{~mL} / \mathrm{min}, 235 \mathrm{~nm}$, $25^{\circ} \mathrm{C}$, retention time $48.365 \mathrm{~min}$ (major) and $56.935 \mathrm{~min}$.

3al. Yellow solid, $43.3 \mathrm{mg}, 43 \%$ yield, $93 \%$ ee. $[\alpha]_{\mathrm{D}^{20}}=-65.9$ (c $=0.22, \mathrm{CH}_{3} \mathrm{CN}$ ). ${ }^{1} \mathrm{H}$ NMR (400 MHz, DMSO-d 6$) \delta 9.69(\mathrm{~s}, 1 \mathrm{H})$, $7.57(\mathrm{~s}, 1 \mathrm{H}), 7.52(\mathrm{~d}, J=8.3 \mathrm{~Hz}, 2 \mathrm{H}), 7.38-7.35(\mathrm{~m}, 2 \mathrm{H}), 7.32(\mathrm{~d}, J$ $=8.1 \mathrm{~Hz}, 2 \mathrm{H}), 7.18-7.08(\mathrm{~m}, 2 \mathrm{H}), 6.99-6.93(\mathrm{~m}, 1 \mathrm{H}), 6.71(\mathrm{~d}, J=$ $15.7 \mathrm{~Hz}, 1 \mathrm{H}), 6.01-5.90(\mathrm{~m}, 1 \mathrm{H}), 3.58(\mathrm{~d}, J=18.5 \mathrm{~Hz}, 1 \mathrm{H}), 3.23$ $(\mathrm{d}, J=18.5 \mathrm{~Hz}, 1 \mathrm{H}), 2.83-2.68(\mathrm{~m}, 2 \mathrm{H}), 2.34(\mathrm{~s}, 3 \mathrm{H}), 2.33(\mathrm{~s}, 3 \mathrm{H})$, 2.29 (s, 3H). ${ }^{13} \mathrm{C}$ NMR (101 MHz, DMSO-d6) $\delta 199.57,148.81$, $147.57,143.50,138.09,137.68,133.95,133.49,131.32,130.99$, 130.02, 128.63, 127.72, 127.61, 127.20 (two C signals overlapped), 126.47, 125.19, 124.23, 110.69, 59.64, 39.31, 37.63, 21.44, 20.88, 19.72. HRMS (ESI): calculated for $\mathrm{C}_{28} \mathrm{H}_{26} \mathrm{~N}_{2} \mathrm{O}_{3} \mathrm{~S}_{2}$ $[\mathrm{M}+\mathrm{H}]^{+}:$503.1458, found: 503.1440. HPLC: Chiralpak AD-H column, hexane $/ i-\mathrm{PrOH}=80 / 20$, flow $=1.0 \mathrm{~mL} / \mathrm{min}, 235 \mathrm{~nm}$, $25^{\circ} \mathrm{C}$, retention time $38.616 \mathrm{~min}$ (major) and $41.683 \mathrm{~min}$.

3ba. Yellow solid, $42.1 \mathrm{mg}, 43 \%$ yield, $93 \%$ ee. $[\alpha]_{\mathrm{D}}^{20}=-61.2$ (c = 0.42, $\mathrm{CH}_{3} \mathrm{CN}$ ). ${ }^{1} \mathrm{H}$ NMR (400 MHz, DMSO-d6) $\delta 9.70(\mathrm{~s}, 1 \mathrm{H})$, 7.86-7.76 (m, 2H), $7.60(\mathrm{~d}, J=7.7 \mathrm{~Hz}, 1 \mathrm{H}), 7.55(\mathrm{~d}, J=8.1 \mathrm{~Hz}$, $2 \mathrm{H}), 7.53(\mathrm{~d}, J=7.0 \mathrm{~Hz}, 1 \mathrm{H}), 7.35(\mathrm{~d}, J=8.1 \mathrm{~Hz}, 2 \mathrm{H}), 7.31(\mathrm{~d}, J=$ $7.8 \mathrm{~Hz}, 1 \mathrm{H}), 7.28-7.20(\mathrm{~m}, 1 \mathrm{H}), 7.12-7.05(\mathrm{~m}, 1 \mathrm{H}), 6.61(\mathrm{~d}, J=$ $15.8 \mathrm{~Hz}, 1 \mathrm{H}), 6.19-6.07(\mathrm{~m}, 1 \mathrm{H}), 3.66(\mathrm{~d}, J=18.6 \mathrm{~Hz}, 1 \mathrm{H}), 3.34$ (d, $J=18.6 \mathrm{~Hz}, 1 \mathrm{H}), 2.84-2.66(\mathrm{~m}, 2 \mathrm{H}), 2.36(\mathrm{~s}, 3 \mathrm{H}) .{ }^{13} \mathrm{C}$ NMR (101 MHz, DMSO-d6) $\delta 199.99,159.65$ (d, $J=248.3 \mathrm{~Hz}$ ), 150.68, 143.51, 138.44, 138.37, 137.06, 133.22, 130.45 (d, $J=3.1 \mathrm{~Hz}$ ), $129.98,129.34$ (d, $J=8.7 \mathrm{~Hz}), 129.15,127.37,127.16,125.60$, $125.23,121.90$ (d, $J=3.1 \mathrm{~Hz}), 121.62$ (d, $J=13.9 \mathrm{~Hz}), 115.41$ (d, $J=21.0 \mathrm{~Hz}), 110.52,59.20,38.96,37.92,21.44 .{ }^{19} \mathrm{~F}$ NMR $(376$ MHz, DMSO-d 6 ) $\delta-118.41$. HRMS (ESI): calculated for $\mathrm{C}_{26} \mathrm{H}_{21} \mathrm{FN}_{2} \mathrm{O}_{3} \mathrm{~S}_{2}\left[\mathrm{M}+\mathrm{NH}_{4}\right]^{+}$: 510.1316, found: 510.1312. HPLC: 
Chiralpak IC column, hexane $/ i-\mathrm{PrOH}=75 / 25$, flow $=1.0$ $\mathrm{mL} / \mathrm{min}, 235 \mathrm{~nm}, 25^{\circ} \mathrm{C}$, retention time $82.949 \mathrm{~min}$ (major) and $92.307 \mathrm{~min}$.

3ca. Yellow solid, $38.9 \mathrm{mg}, 38 \%$ yield, $94 \%$ ee. $[\alpha]_{\mathrm{D}^{20}}=-46.7$ (c = 0.39, $\mathrm{CH}_{3} \mathrm{CN}$ ). ${ }^{1} \mathrm{H}$ NMR (400 MHz, DMSO-d 6$) \delta 9.94(\mathrm{~s}, 1 \mathrm{H})$, 7.84-7.77 (m, 2H), 7.60 (d, $J=7.6 \mathrm{~Hz}, 1 \mathrm{H}), 7.57-7.51(\mathrm{~m}, 3 \mathrm{H})$, $7.42(\mathrm{~d}, J=8.6 \mathrm{~Hz}, 1 \mathrm{H}), 7.36(\mathrm{~d}, J=8.1 \mathrm{~Hz}, 2 \mathrm{H}), 7.21(\mathrm{dd}, J=8.5$, $2.2 \mathrm{~Hz}, 1 \mathrm{H}), 7.04$ (d, $J=2.2 \mathrm{~Hz}, 1 \mathrm{H}), 6.64$ (d, $J=15.7 \mathrm{~Hz}, 1 \mathrm{H}$ ), 6.11-5.99 (m, 1H), $3.66(\mathrm{~d}, J=18.6 \mathrm{~Hz}, 1 \mathrm{H}), 3.33(\mathrm{~d}, J=18.1 \mathrm{~Hz}$, $1 \mathrm{H}), 2.87-2.71(\mathrm{~m}, 2 \mathrm{H}), 2.36(\mathrm{~s}, 3 \mathrm{H}) .{ }^{13} \mathrm{C} \mathrm{NMR}(101 \mathrm{MHz}$, DMSO-d 6$) \delta 200.00,150.68,143.89,137.21,137.06,135.17$, 133.22, 132.35, 132.05, 130.18, 129.71, 129.15, 128.13, 127.35, 127.19, 127.04, 126.80, 125.43, 125.23, 110.54, 59.28, 38.89, 37.93, 21.46. HRMS (ESI): calculated for $\mathrm{C}_{26} \mathrm{H}_{21} \mathrm{ClN}_{2} \mathrm{O}_{3} \mathrm{~S}_{2}$ [M+NH4 $]^{+}:$526.1020, found: 526.1074. HPLC: Chiralpak IC column, hexane $/ i-\mathrm{PrOH}=70 / 30$, flow $=1.0 \mathrm{~mL} / \mathrm{min}, 235 \mathrm{~nm}$, $25^{\circ} \mathrm{C}$, retention time $100.910 \mathrm{~min}$ (major) and $77.442 \mathrm{~min}$.

3da. Yellow solid. $65.8 \mathrm{mg}, 67 \%$ yield, $93 \%$ ee. $[\alpha]_{\mathrm{D}^{20}}=-65.5$ (c $=0.66, \mathrm{CH}_{3} \mathrm{CN}$ ). ${ }^{1} \mathrm{H}$ NMR $\left(400 \mathrm{MHz}, \mathrm{DMSO}-\mathrm{d}_{6}\right) \delta 9.63(\mathrm{~s}, 1 \mathrm{H})$, 7.84-7.77 (m, 2H), 7.60 (d, $J=7.6 \mathrm{~Hz}, 1 \mathrm{H}), 7.55(\mathrm{~d}, J=7.2 \mathrm{~Hz}$, $1 \mathrm{H}), 7.53$ (d, $J=8.0 \mathrm{~Hz}, 2 \mathrm{H}), 7.33(\mathrm{~d}, J=8.1 \mathrm{~Hz}, 2 \mathrm{H}), 7.25(\mathrm{~d}, J=$ $8.0 \mathrm{~Hz}, 1 \mathrm{H}), 6.95(\mathrm{~d}, J=8.0 \mathrm{~Hz}, 1 \mathrm{H}), 6.83(\mathrm{~s}, 1 \mathrm{H}), 6.62$ (d, $J=15.6$ $\mathrm{Hz}, 1 \mathrm{H}), 5.95-5.84(\mathrm{~m}, 1 \mathrm{H}), 3.65$ (d, $J=18.6 \mathrm{~Hz}, 1 \mathrm{H}), 3.32$ (d, $J=$ $18.7 \mathrm{~Hz}, 1 \mathrm{H}), 2.83-2.67(\mathrm{~m}, 2 \mathrm{H}), 2.35$ (s, 3H), 2.17 (s, 3H). ${ }^{13} \mathrm{C}$ NMR (101 MHz, DMSO-d 6$) \delta 200.14,150.74,143.46,138.12$, $137.74,137.04,133.78,133.30,130.86,130.65,129.97,129.12$, 128.28, 128.01, 127.34, 127.21, 126.22, 125.19, 123.08, 110.59, 59.39, 39.08, 37.97, 21.44, 21.07. HRMS (ESI): calculated for $\mathrm{C}_{27} \mathrm{H}_{24} \mathrm{~N}_{2} \mathrm{O}_{3} \mathrm{~S}_{2}[\mathrm{M}+\mathrm{Na}]+:$ 511.1121, found: 511.1124. HPLC: Chiralpak IC column, hexane $/ i-\mathrm{PrOH}=70 / 30$, flow $=1.0 \mathrm{~mL} / \mathrm{min}$, $235 \mathrm{~nm}, 25^{\circ} \mathrm{C}$, retention time $103.819 \mathrm{~min}$ (major) and 93.315 $\min$.

3ea. Yellow solid, $35.2 \mathrm{mg}, 36 \%$ yield, $88 \%$ ee. $[\alpha]_{\mathrm{D}}^{20}=-70.9$ (c $=0.35, \mathrm{CH}_{3} \mathrm{CN}$ ). ${ }^{1} \mathrm{H}$ NMR (400 MHz, DMSO-d 6$) \delta 9.70(\mathrm{~s}, 1 \mathrm{H})$, $7.86-7.76(\mathrm{~m}, 2 \mathrm{H}), 7.61(\mathrm{~d}, J=7.6 \mathrm{~Hz}, 1 \mathrm{H}), 7.56(\mathrm{~d}, J=7.4 \mathrm{~Hz}$, $1 \mathrm{H}), 7.51(\mathrm{~d}, J=8.3 \mathrm{~Hz}, 2 \mathrm{H}), 7.34(\mathrm{~d}, J=8.1 \mathrm{~Hz}, 2 \mathrm{H}), 7.28$ (dd, $J=$ $10.2,2.9 \mathrm{~Hz}, 1 \mathrm{H}), 7.03(\mathrm{td}, J=8.4,2.9 \mathrm{~Hz}, 1 \mathrm{H}), 6.95(\mathrm{dd}, J=8.8$, $5.6 \mathrm{~Hz}, 1 \mathrm{H}), 6.65$ (d, $J=15.4 \mathrm{~Hz}, 1 \mathrm{H}), 6.23-6.07(\mathrm{~m}, 1 \mathrm{H}), 3.67$ (d, $J=18.7 \mathrm{~Hz}, 1 \mathrm{H}), 3.34(\mathrm{~d}, J=18.6 \mathrm{~Hz}, 1 \mathrm{H}), 2.87-2.70(\mathrm{~m}, 2 \mathrm{H})$, 2.36 (s, 3H). ${ }^{13}$ C NMR (101 MHz, DMSO-d 6$) \delta 199.93,161.13$ (d, $J=243.4 \mathrm{~Hz}$ ), 150.68, 143.62, 137.36, 137.02, 136.38 (d, $J=8.5$ $\mathrm{Hz}), 133.24,130.47$ (d, $J=9.0 \mathrm{~Hz}), 130.13$ (d, $J=2.6 \mathrm{~Hz}$ ), 130.08, $130.03,129.12,127.34,127.25,126.05,125.25,115.49$ (d, $J=$ $22.9 \mathrm{~Hz}$ ), 112.60 (d, $J=23.4 \mathrm{~Hz}$ ), 110.56, 59.31, 38.82, 37.91, 21.46. ${ }^{19} \mathrm{~F}$ NMR (376 MHz, DMSO-d6) $\delta-114.93$. HRMS (ESI): calculated for $\mathrm{C}_{26} \mathrm{H}_{21} \mathrm{FN}_{2} \mathrm{O}_{3} \mathrm{~S}_{2} \quad\left[\mathrm{M}+\mathrm{NH}_{4}\right]^{+}:$510.1316, found: 510.1313. HPLC: Chiralpak IA column, hexane $/ i-\mathrm{PrOH}=80 / 20$, flow $=1.0 \mathrm{~mL} / \mathrm{min}, 235 \mathrm{~nm}, 25^{\circ} \mathrm{C}$, retention time $20.475 \mathrm{~min}$ (major) and $24.776 \mathrm{~min}$.

3fa. Yellow solid, $29.5 \mathrm{mg}, 29 \%$ yield, $89 \%$ ee. $[\alpha]_{\mathrm{D}}{ }^{20}=-47.7$ (c $=0.30, \mathrm{CH}_{3} \mathrm{CN}$ ). ${ }^{1} \mathrm{H}$ NMR (400 MHz, DMSO-d 6$) \delta 9.80(\mathrm{~s}, 1 \mathrm{H})$, $7.81(\mathrm{t}, J=8.3 \mathrm{~Hz}, 2 \mathrm{H}), 7.61(\mathrm{~d}, J=7.6 \mathrm{~Hz}, 1 \mathrm{H}), 7.56(\mathrm{~d}, J=7.5 \mathrm{~Hz}$, 1H), $7.53(\mathrm{~d}, J=8.2 \mathrm{~Hz}, 2 \mathrm{H}), 7.46(\mathrm{~d}, J=2.4 \mathrm{~Hz}, 1 \mathrm{H}), 7.34(\mathrm{~d}, J=$ $8.1 \mathrm{~Hz}, 2 \mathrm{H}), 7.24(\mathrm{dd}, J=8.6,2.4 \mathrm{~Hz}, 1 \mathrm{H}), 7.00(\mathrm{~d}, J=8.6 \mathrm{~Hz}, 1 \mathrm{H})$, $6.64(\mathrm{~d}, J=15.7 \mathrm{~Hz}, 1 \mathrm{H}), 6.18-6.07(\mathrm{~m}, 1 \mathrm{H}), 3.68$ (d, $J=18.6 \mathrm{~Hz}$, 1H), 3.35 (d, $J=18.4 \mathrm{~Hz}, 1 \mathrm{H}), 2.87-2.69(\mathrm{~m}, 2 \mathrm{H}), 2.35$ (s, 3H). ${ }^{13} \mathrm{C}$ NMR (101 MHz, DMSO-d6) $\delta 199.96,150.70,143.74,137.33$,
$137.04,135.47,133.24,132.83,131.81,130.14,129.63,129.43$, 129.13, 128.42, 127.33, 127.20, 126.35, 126.10, 125.24, 110.57, 59.36, 38.89, 37.96, 21.46. HRMS (ESI): calculated for $\mathrm{C}_{26} \mathrm{H}_{21} \mathrm{ClN}_{2} \mathrm{O}_{3} \mathrm{~S}_{2}\left[\mathrm{M}+\mathrm{NH}_{4}\right]^{+}:$526.1020, found: 526.1013. HPLC: Chiralpak OJ-H column, hexane $/ i-\mathrm{PrOH}=85 / 15$, flow $=1.0$ $\mathrm{mL} / \mathrm{min}, 235 \mathrm{~nm}, 25^{\circ} \mathrm{C}$, retention time $85.255 \mathrm{~min}$ (major) and $106.796 \mathrm{~min}$.

3ga. Yellow solid, $28.0 \mathrm{mg}, 25 \%$ yield, $88 \%$ ee. $[\alpha]_{\mathrm{D}}{ }^{20}=-32.5$ (c $=0.28, \mathrm{CH}_{3} \mathrm{CN}$ ). ${ }^{1} \mathrm{H}$ NMR $\left(400 \mathrm{MHz}, \mathrm{CDCl}_{3}\right) \delta 7.87(\mathrm{~d}, J=7.7$ $\mathrm{Hz}, 1 \mathrm{H}), 7.70(\mathrm{td}, J=7.6,1.1 \mathrm{~Hz}, 1 \mathrm{H}), 7.61(\mathrm{~d}, J=8.3 \mathrm{~Hz}, 2 \mathrm{H})$, 7.49 (d, $J=7.4 \mathrm{~Hz}, 1 \mathrm{H}$ ), 7.45 (d, $J=7.7 \mathrm{~Hz}, 1 \mathrm{H}), 7.29-7.26$ (m, 1H), $7.25(\mathrm{~s}, 3 \mathrm{H}), 7.06(\mathrm{~d}, J=8.4 \mathrm{~Hz}, 1 \mathrm{H}), 6.75(\mathrm{~s}, 1 \mathrm{H}), 6.41$ (d, $J=$ $15.6 \mathrm{~Hz}, 1 \mathrm{H}), 5.92-5.81(\mathrm{~m}, 1 \mathrm{H}), 3.54$ (d, $J=18.2 \mathrm{~Hz}, 1 \mathrm{H}), 3.33$ (d, $J=18.2 \mathrm{~Hz}, 1 \mathrm{H}), 2.93-2.81(\mathrm{~m}, 2 \mathrm{H}), 2.41(\mathrm{~s}, 3 \mathrm{H}) .{ }^{13} \mathrm{C} \mathrm{NMR}$ (101 MHz, $\left.\mathrm{CDCl}_{3}\right) \delta 199.40,149.64,144.31,136.70,136.14$, 134.10, 133.34, 132.25, 131.56, 129.95, 129.85(two carbon signals overlap), 128.91, 127.27, 127.21, 127.09, 126.56, 125.57, 120.28, 109.74, 59.27, 40.15, 39.01, 21.62. HRMS (ESI): calculated for $\mathrm{C}_{26} \mathrm{H}_{21} \mathrm{BrN}_{2} \mathrm{O}_{3} \mathrm{~S}_{2}\left[\mathrm{M}+\mathrm{NH}_{4}\right]^{+}: 570.0515$, found: 570.0525. HPLC: Chiralpak IC column, hexane $/ i-\mathrm{PrOH}=80 / 20$, flow $=1.0 \mathrm{~mL} / \mathrm{min}, 235 \mathrm{~nm}, 25^{\circ} \mathrm{C}$, retention time $129.266 \mathrm{~min}$ (major) and $121.198 \mathrm{~min}$.

3ha. Yellow solid. $72.2 \mathrm{mg}, 74 \%$ yield, $90 \%$ ee. $[\alpha]_{\mathrm{D}}{ }^{20}=-50.8$ (c $\left.=0.72, \mathrm{CH}_{3} \mathrm{CN}\right) .{ }^{1} \mathrm{H}$ NMR (400 MHz, DMSO-d $) \delta 9.57(\mathrm{~s}, 1 \mathrm{H})$, 7.85-7.77 (m, 2H), 7.60 (d, $J=7.7 \mathrm{~Hz}, 1 \mathrm{H}), 7.58-7.50(\mathrm{~m}, 3 \mathrm{H})$, $7.32(\mathrm{~d}, J=8.1 \mathrm{~Hz}, 2 \mathrm{H}), 7.18(\mathrm{~s}, 1 \mathrm{H}), 6.96(\mathrm{dd}, J=8.1,1.4 \mathrm{~Hz}, 1 \mathrm{H})$, $6.84(\mathrm{~d}, J=8.1 \mathrm{~Hz}, 1 \mathrm{H}), 6.69$ (d, $J=15.7 \mathrm{~Hz}, 1 \mathrm{H}), 6.04-5.90(\mathrm{~m}$, $1 \mathrm{H}), 3.68(\mathrm{~d}, J=18.6 \mathrm{~Hz}, 1 \mathrm{H}), 3.34(\mathrm{~d}, J=18.7 \mathrm{~Hz}, 1 \mathrm{H}), 2.88-$ $2.70(\mathrm{~m}, 2 \mathrm{H}), 2.35$ (s, 3H), $2.20(\mathrm{~s}, 3 \mathrm{H}) .{ }^{13} \mathrm{C}$ NMR $(101 \mathrm{MHz}$, DMSO-d6) $\delta$ 200.12, 150.74, 143.41, 137.72, 137.04, 136.62, 133.59, 133.32, 131.41, 131.16, 129.98, 129.33, 129.12, 127.95, 127.35, 127.25, 126.76, 125.22, 123.86, 110.59, 59.40, 39.06, 37.96, 21.45, 20.97. HRMS (ESI): calculated for $\mathrm{C}_{27} \mathrm{H}_{24} \mathrm{~N}_{2} \mathrm{O}_{3} \mathrm{~S}_{2}$ $\left[\mathrm{M}+\mathrm{NH}_{4}\right]^{+}:$506.1567, found: 506.1575. HPLC: Chiralpak IC column, hexane $/ i-\mathrm{PrOH}=80 / 20$, flow $=1.0 \mathrm{~mL} / \mathrm{min}, 235 \mathrm{~nm}$, $25^{\circ} \mathrm{C}$, retention time $181.822 \mathrm{~min}$ (major) and $171.427 \mathrm{~min}$.

3ia. Yellow solid, $65.6 \mathrm{mg}, 65 \%$ yield, $92 \%$ ee. $[\alpha]_{\mathrm{D}}^{20}=-60.0$ (c = 0.66, $\mathrm{CH}_{3} \mathrm{CN}$ ). ${ }^{1} \mathrm{H}$ NMR $\left(400 \mathrm{MHz}, \mathrm{DMSO}-\mathrm{d}_{6}\right) \delta 9.47(\mathrm{~s}, 1 \mathrm{H})$, $7.81(\mathrm{dd}, J=12.2,7.6 \mathrm{~Hz}, 2 \mathrm{H}), 7.60(\mathrm{~d}, J=7.7 \mathrm{~Hz}, 1 \mathrm{H}), 7.55(\mathrm{~d}, J=$ $7.5 \mathrm{~Hz}, 1 \mathrm{H}), 7.51$ (d, $J=8.3 \mathrm{~Hz}, 2 \mathrm{H}), 7.33(\mathrm{~d}, J=8.2 \mathrm{~Hz}, 2 \mathrm{H}), 6.87$ $(\mathrm{d}, J=2.8 \mathrm{~Hz}, 1 \mathrm{H}), 6.82(\mathrm{~d}, J=8.8 \mathrm{~Hz}, 1 \mathrm{H}), 6.75(\mathrm{dd}, J=8.8,2.8$ $\mathrm{Hz}, 1 \mathrm{H}), 6.65$ (d, $J=15.7 \mathrm{~Hz}, 1 \mathrm{H}), 6.08-5.96(\mathrm{~m}, 1 \mathrm{H}), 3.69$ (s, $3 \mathrm{H}), 3.68(\mathrm{~d}, J=18.5 \mathrm{~Hz}, 3 \mathrm{H}), 3.35$ (d, $J=18.6 \mathrm{~Hz}, 1 \mathrm{H}), 2.83-2.70$ $(\mathrm{m}, J=14.4,7.1 \mathrm{~Hz}, 2 \mathrm{H}), 2.36(\mathrm{~s}, 3 \mathrm{H}) .{ }^{13} \mathrm{C}$ NMR $(101 \mathrm{MHz}$, DMSO-d6) $\delta$ 200.12, 158.41, 150.77, 143.36, 137.70, 137.05, 135.68, 133.32, 131.15, 130.06, 129.97, 129.11, 127.32, 127.29, 126.75, 125.19, 124.41, 114.51, 110.76, 110.64, 59.41, 55.73, 39.17, 38.02, 21.45. HRMS (ESI): calculated for $\mathrm{C}_{27} \mathrm{H}_{24} \mathrm{~N}_{2} \mathrm{O}_{4} \mathrm{~S}_{2}$ $[\mathrm{M}+\mathrm{H}]^{+}:$505.1250, found: 505.1252. HPLC: Chiralpak IC column, hexane $/ \mathrm{i}-\mathrm{PrOH}=80 / 20$, flow $=1.0 \mathrm{~mL} / \mathrm{min}, 235 \mathrm{~nm}, 25$ ${ }^{\circ} \mathrm{C}$, retention time $34.550 \mathrm{~min}$ (major) and $41.316 \mathrm{~min}$.

3ja. Yellow solid, $40.7 \mathrm{mg}, 41 \%$ yield, $86 \%$ ee. $[\alpha]_{\mathrm{D}^{20}}=-54.5$ (c $\left.=0.20, \mathrm{CH}_{3} \mathrm{CN}\right) .{ }^{1} \mathrm{H}$ NMR $\left(400 \mathrm{MHz}, \mathrm{DMSO}-\mathrm{d}_{6}\right) \delta 9.85(\mathrm{~s}, 1 \mathrm{H})$, 7.81 (dd, $J=12.9,7.5 \mathrm{~Hz}, 2 \mathrm{H}), 7.61(\mathrm{~d}, J=7.7 \mathrm{~Hz}, 1 \mathrm{H}), 7.59-7.51$ (m, 3H), 7.36 (d, $J=8.1 \mathrm{~Hz}, 2 \mathrm{H}), 7.19$ (dd, $J=14.4,8.1 \mathrm{~Hz}, 1 \mathrm{H}$ ), $7.04(\mathrm{dd}, J=10.5,8.7 \mathrm{~Hz}, 1 \mathrm{H}), 6.87(\mathrm{~d}, J=8.0 \mathrm{~Hz}, 1 \mathrm{H}), 6.48(\mathrm{~d}, J=$ $16.1 \mathrm{~Hz}, 1 \mathrm{H}), 6.11-5.97$ (m, 1H), 3.65 (d, $J=18.6 \mathrm{~Hz}, 1 \mathrm{H}), 3.32$ 
(d, $J=18.7 \mathrm{~Hz}, 1 \mathrm{H}), 2.92-2.74(\mathrm{~m}, 2 \mathrm{H}), 2.37(\mathrm{~s}, 3 \mathrm{H}) .{ }^{13} \mathrm{C}$ NMR (101 MHz, DMSO-d 6 ) $\delta 200.10,160.68$ (d, $J=248.5 \mathrm{~Hz}$ ), 150.68, 143.79, 137.47, 137.08, 136.13 (d, $J=5.7 \mathrm{~Hz}), 133.23,130.14$, $129.61(\mathrm{~d}, J=12.4 \mathrm{~Hz}), 129.16,128.94$ (d, $J=10.5 \mathrm{~Hz}$ ), 127.36, $127.20,125.47,125.19,123.24,121.51$ (d, $J=13.1 \mathrm{~Hz}), 114.55$ (d, $J=23.2 \mathrm{~Hz}), 110.48,58.90,39.78,37.82,21.46 .{ }^{19} \mathrm{~F}$ NMR (376 MHz, DMSO-d 6 ) $\delta-113.01$. HRMS (ESI): calculated for $\mathrm{C}_{26} \mathrm{H}_{21} \mathrm{FN}_{2} \mathrm{O}_{3} \mathrm{~S}_{2}\left[\mathrm{M}+\mathrm{NH}_{4}\right]^{+}:$510.1316, found: 510.1313. HPLC: Chiralpak IC column, hexane $/ i-\mathrm{PrOH}=75 / 25$, flow $=1.0$ $\mathrm{mL} / \mathrm{min}, 235 \mathrm{~nm}, 25^{\circ} \mathrm{C}$, retention time $138.241 \mathrm{~min}$ (major) and $128.873 \mathrm{~min}$.

9aa. White solid, $32.6 \mathrm{mg}, 71 \%$ yield, $80 \%$ ee. $[\alpha]_{\mathrm{D}^{20}}=-38.6$ (c $\left.=0.35, \mathrm{CH}_{3} \mathrm{CN}\right) .{ }^{1} \mathrm{H}$ NMR $\left(400 \mathrm{MHz}, \mathrm{CDCl}_{3}\right) \delta 7.78(\mathrm{~d}, J=7.6$ $\mathrm{Hz}, 1 \mathrm{H}), 7.67-7.57(\mathrm{~m}, 3 \mathrm{H}), 7.48(\mathrm{~d}, J=7.6 \mathrm{~Hz}, 1 \mathrm{H}), 7.41$ (t, $J=$ $7.4 \mathrm{~Hz}, 1 \mathrm{H}), 7.25-7.17(\mathrm{~m}, 3 \mathrm{H}), 7.16-7.10(\mathrm{~m}, 1 \mathrm{H}), 7.05$ (d, $J=$ $4.0 \mathrm{~Hz}, 2 \mathrm{H}), 6.62(\mathrm{~s}, 1 \mathrm{H}), 6.31(\mathrm{~d}, J=15.6 \mathrm{~Hz}, 1 \mathrm{H}), 5.80-5.64(\mathrm{~m}$, 1H), 3.73 (d, $J=17.7 \mathrm{~Hz}, 1 \mathrm{H}), 2.99(\mathrm{~d}, J=17.7 \mathrm{~Hz}, 1 \mathrm{H}), 2.83$ (dd, $J$ = 14.1, $7.9 \mathrm{~Hz}, 1 \mathrm{H}), 2.74(\mathrm{dd}, J=14.1,6.8 \mathrm{~Hz}, 1 \mathrm{H}), 2.39(\mathrm{~s}, 3 \mathrm{H})$, 2.24 (s, 3H). ${ }^{13} \mathrm{C}$ NMR (101 MHz, $\left.\mathrm{CDCl}_{3}\right) \delta 203.47,202.99$, $153.18,143.86,136.57,135.75,135.28,133.03,132.28,129.63$, 129.08, 128.47, 128.33, 127.97, 127.26, 127.19, 126.68, 126.45, 125.04, 124.67, 68.27, 38.67, 34.36, 26.25, 21.57. HRMS (ESI): calculated for $\mathrm{C}_{27} \mathrm{H}_{25} \mathrm{NO}_{4} \mathrm{~S}[\mathrm{M}+\mathrm{H}]^{+}: 460.1577$, found: 460.1579 . HPLC: Chiralpak AD-H column, hexane $/ i-\mathrm{PrOH}=70 / 30$, flow $=$ $1.0 \mathrm{~mL} / \mathrm{min}, 235 \mathrm{~nm}, 25^{\circ} \mathrm{C}$, retention time 19.327 min (major) and $24.083 \mathrm{~min}$.

9ab. White solid, $14.4 \mathrm{mg}, 59 \%$ yield, $77 \%$ ee. $[\alpha]_{\mathrm{D}^{20}}=-51.7$ (c = 0.29, $\mathrm{CH}_{3} \mathrm{CN}$ ). ${ }^{1} \mathrm{H}$ NMR (400 MHz, $\left.\mathrm{CDCl}_{3}\right) \delta 7.81(\mathrm{~d}, J=7.7$ $\mathrm{Hz}, 1 \mathrm{H}), 7.67-7.57(\mathrm{~m}, 3 \mathrm{H}), 7.49(\mathrm{~d}, J=7.7 \mathrm{~Hz}, 1 \mathrm{H}), 7.42$ (t, $J=$ $7.4 \mathrm{~Hz}, 1 \mathrm{H}), 7.22(\mathrm{t}, J=7.3 \mathrm{~Hz}, 3 \mathrm{H}), 7.12(\mathrm{t}, J=7.1 \mathrm{~Hz}, 1 \mathrm{H}), 7.07-$ $6.94(\mathrm{~m}, 2 \mathrm{H}), 6.57(\mathrm{~s}, 1 \mathrm{H}), 6.21(\mathrm{~d}, J=15.6 \mathrm{~Hz}, 1 \mathrm{H}), 5.75-5.62$ (m, 1H), 3.64 (d, $J=17.7 \mathrm{~Hz}, 1 \mathrm{H}), 3.09-2.96(\mathrm{~m}, 2 \mathrm{H}), 2.79$ (dd, $J$ = 14.0,6.9 Hz, 1H), $2.72(\mathrm{dd}, J=14.0,7.9 \mathrm{~Hz}, 1 \mathrm{H}), 2.39(\mathrm{~s}, 3 \mathrm{H})$, $1.04(\mathrm{~d}, J=6.7 \mathrm{~Hz}, 3 \mathrm{H}), 0.97(\mathrm{~d}, J=6.7 \mathrm{~Hz}, 3 \mathrm{H}) .{ }^{13} \mathrm{C}$ NMR $(101$ $\left.\mathrm{MHz}, \mathrm{CDCl}_{3}\right) \delta 210.32,203.33,152.95,143.84,136.59,136.00$, 135.66, 133.06, 132.13, 129.62, 129.07, 128.97, 128.25, 128.04, 127.28, 127.26, 126.69, 126.27, 124.67, 124.55, 67.73, 38.29, 36.51, 34.57, 21.57, 20.18, 19.74. HRMS (ESI): calculated for $\mathrm{C}_{29} \mathrm{H}_{29} \mathrm{NO}_{4} \mathrm{~S}[\mathrm{M}+\mathrm{H}]^{+}:$488.1890, found: 488.1894. HPLC: Chiralpak $\mathrm{AD}-\mathrm{H}$ column, hexane $/ i-\mathrm{PrOH}=70 / 30$, flow $=1.0$ $\mathrm{mL} / \mathrm{min}, 235 \mathrm{~nm}, 25^{\circ} \mathrm{C}$, retention time $18.132 \mathrm{~min}$ (major) and $23.257 \mathrm{~min}$.

9ac. White solid, $9.6 \mathrm{mg}$, 37\% yield, $73 \%$ ee. $[\alpha]_{\mathrm{D}}{ }^{20}=-94.7$ (c $\left.=0.17, \mathrm{CH}_{3} \mathrm{CN}\right) .{ }^{1} \mathrm{H}$ NMR $\left(400 \mathrm{MHz}, \mathrm{CDCl}_{3}\right) \delta 7.95(\mathrm{~d}, J=7.8 \mathrm{~Hz}$, $1 \mathrm{H}), 7.71(\mathrm{t}, J=7.4 \mathrm{~Hz}, 1 \mathrm{H}), 7.63-7.45(\mathrm{~m}, 7 \mathrm{H}), 7.31(\mathrm{t}, J=7.6$ $\mathrm{Hz}, 2 \mathrm{H}), 7.25(\mathrm{~d}, J=9.8 \mathrm{~Hz}, 1 \mathrm{H}), 7.18(\mathrm{~d}, J=8.0 \mathrm{~Hz}, 2 \mathrm{H}), 7.12(\mathrm{t}, \mathrm{J}$ $=7.8 \mathrm{~Hz}, 1 \mathrm{H}), 7.00(\mathrm{t}, J=7.5 \mathrm{~Hz}, 1 \mathrm{H}), 6.85(\mathrm{~d}, J=7.7 \mathrm{~Hz}, 1 \mathrm{H})$, $6.45(\mathrm{~s}, 1 \mathrm{H}), 6.05(\mathrm{~d}, J=15.6 \mathrm{~Hz}, 1 \mathrm{H}), 5.82-5.68(\mathrm{~m}, 1 \mathrm{H}), 3.68(\mathrm{~d}$, $J=17.9 \mathrm{~Hz}, 1 \mathrm{H}$ ), 3.23 (d, $J=17.7 \mathrm{~Hz}, 1 \mathrm{H}), 2.88$ (dd, $J=13.7,6.9$ $\mathrm{Hz}, 1 \mathrm{H}), 2.78$ (dd, $J=13.7,7.9 \mathrm{~Hz}, 1 \mathrm{H}), 2.38$ (s, 3H). ${ }^{13} \mathrm{C}$ NMR $\left(101 \mathrm{MHz}, \mathrm{CDCl}_{3}\right) \delta 203.67,197.63,152.01,143.79,136.63$, $136.01,135.76,135.18,133.08,132.97,132.26,129.63,129.58$, $129.05,128.62,128.44,128.21,128.17,127.33,127.20,127.05$, $126.16,125.13,124.57,65.86,39.88,37.98,21.56$. HRMS (ESI): calculated for $\mathrm{C}_{32} \mathrm{H}_{27} \mathrm{NO}_{4} \mathrm{~S}[\mathrm{M}+\mathrm{H}]+$ : 522.1734 , found: 522.1739 . HPLC: Chiralpak AD-H column, hexane $/ i-\mathrm{PrOH}=70 / 30$, flow $=$ $1.0 \mathrm{~mL} / \mathrm{min}, 235 \mathrm{~nm}, 25^{\circ} \mathrm{C}$, retention time $61.748 \mathrm{~min}$ (major) and $81.086 \mathrm{~min}$.

9ad. White solid, $13.6 \mathrm{mg}, 53 \%$ yield, $76 \%$ ee. $[\alpha]_{\mathrm{D}^{20}}=-28.2$ (c $=0.27, \mathrm{CH}_{3} \mathrm{CN}$ ). ${ }^{1} \mathrm{H}$ NMR $\left(400 \mathrm{MHz}, \mathrm{CDCl}_{3}\right) \delta 7.80(\mathrm{~d}, J=7.7$ $\mathrm{Hz}, 1 \mathrm{H}), 7.67-7.57$ (m, 3H), 7.46 (d, $J=7.6 \mathrm{~Hz}, 1 \mathrm{H}), 7.40$ (t, $J=$ $7.4 \mathrm{~Hz}, 1 \mathrm{H}), 7.25$ (d, $J=8.1 \mathrm{~Hz}, 1 \mathrm{H}), 7.21(\mathrm{~d}, J=8.0 \mathrm{~Hz}, 2 \mathrm{H}), 7.13$ $(\mathrm{t}, J=7.4 \mathrm{~Hz}, 1 \mathrm{H}), 7.08-6.97(\mathrm{~m}, 2 \mathrm{H}), 6.52(\mathrm{~s}, 1 \mathrm{H}), 6.22(\mathrm{~d}, J=$ $15.6 \mathrm{~Hz}, 1 \mathrm{H}$ ), 5.86-5.73 (m, 1H), 3.56 (d, $J=17.3 \mathrm{~Hz}, 1 \mathrm{H}), 3.08$ (d, $J=17.3 \mathrm{~Hz}, 1 \mathrm{H}), 2.78(\mathrm{dd}, J=13.9,7.6 \mathrm{~Hz}, 1 \mathrm{H}), 2.64$ (dd, $J=$ 13.9, $7.1 \mathrm{~Hz}, 1 \mathrm{H}), 2.39$ (s, 3H), 1.39 (s, 9H). ${ }^{13} \mathrm{C}$ NMR (101 MHz, $\mathrm{CDCl}_{3}$ ) $\delta 202.53,169.85,152.96,143.82,136.59,135.34$ (two carbon signals overlapped), 133.09, 132.21, 129.61, 128.74, $128.19,127.78,127.28,127.25,126.44,126.24,124.70,124.61$, 82.14, 60.82, 38.18, 36.69, 27.85, 21.57. HRMS (ESI): calculated for $\mathrm{C}_{30} \mathrm{H}_{31} \mathrm{NO}_{5} \mathrm{~S}\left[\mathrm{M}+\mathrm{NH}_{4}\right]^{+}$: 535.2261, found: 535.2267. HPLC: Chiralpak AD-H column, hexane $/ i-\mathrm{PrOH}=85 / 15$, flow $=1.0$ $\mathrm{mL} / \mathrm{min}, 235 \mathrm{~nm}, 25^{\circ} \mathrm{C}$, retention time $71.413 \mathrm{~min}$ (major) and $75.306 \mathrm{~min}$.

\section{Results and discussion}

Vinyl benzoxazinanone 1a and $\alpha$-thiocyanato ketone 2a

\section{Table 1}

Optimization of reaction conditions ${ }^{a}$.

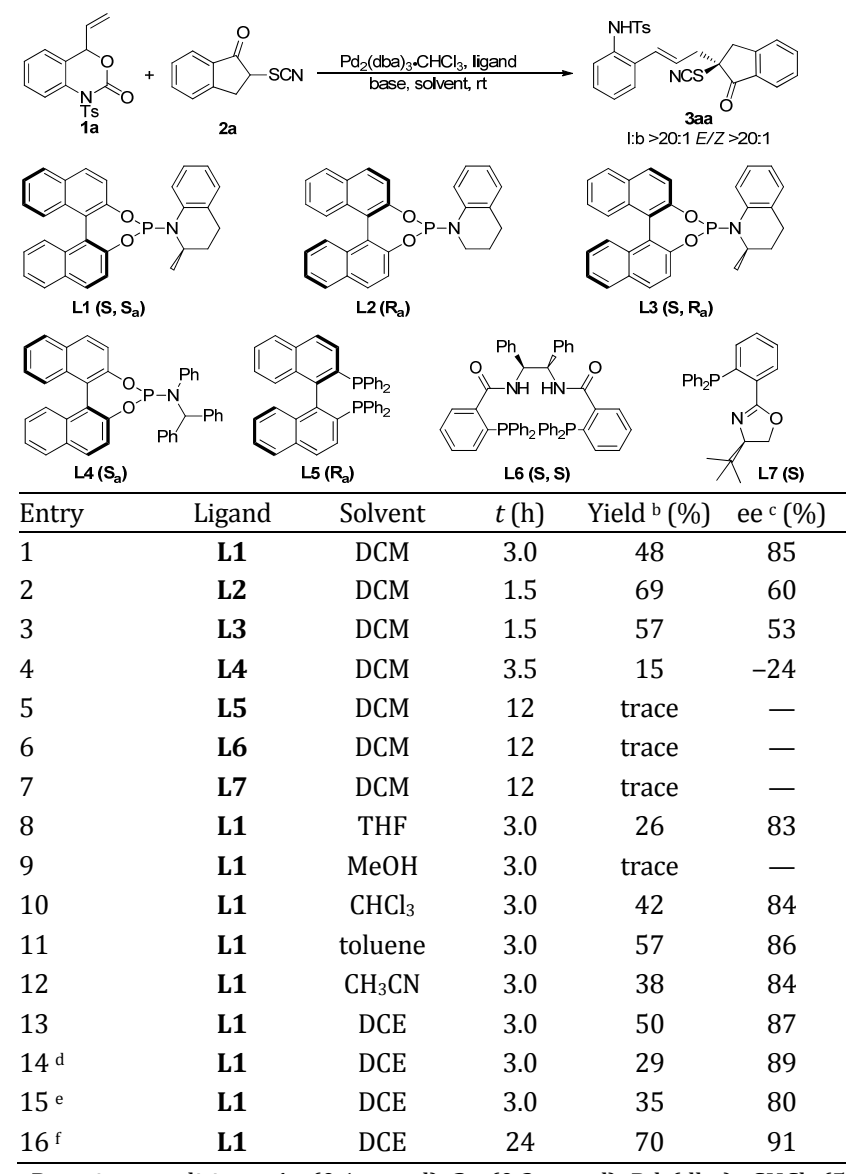

a Reaction conditions: 1a $(0.1 \mathrm{mmol}), \mathbf{2 a}(0.2 \mathrm{mmol}), \mathrm{Pd}_{2}\left(\mathrm{dba}_{3} \cdot \mathrm{CHCl}_{3}(5\right.$ mol\%), chiral ligand (11 mol\%) and $\mathrm{K}_{2} \mathrm{CO}_{3}$ (2.0 equiv.) in $1 \mathrm{~mL}$ solvent at room temperature for $4 \mathrm{~h}$. ${ }^{\mathrm{b}}$ Isolated yield. c Determined by chiral HPLC analysis. ${ }^{d} \mathrm{Na}_{2} \mathrm{CO}_{3}$ instead of $\mathrm{K}_{2} \mathrm{CO}_{3}$. ${ }^{e} \mathrm{Cs}_{2} \mathrm{CO}_{3}$ instead of $\mathrm{K}_{2} \mathrm{CO}_{3}$. ${ }^{\mathrm{f}}$ Performed at $0{ }^{\circ} \mathrm{C}$ for $24 \mathrm{~h}$. DCM: dichloromethane; THF: tetrahydrofuran; DCE: 1,2-dichloroethane. 
were employed as model substrates to conduct the catalytic AAA (Table 1). Firstly, we made a detailed evaluation on the chiral ligands (Table 1, entries 1-7). To our delight, the target linear selective product, 3aa was successfully obtained in a moderate yield and a satisfying enantioselectivity of $85 \%$ ee when using BINOL-based phosphoramidite $\mathbf{L 1}$ as ligand (Table 1 , entry 1) $[48,49]$. Neither branch-selective product nor $Z$-isomer of 3aa was detected based on crude ${ }^{1} \mathrm{H}$ NMR analysis. Other structurally similar ligands L2-L4 gave poor enantioselectivities, despite of that both $\mathbf{L} 2$ and $\mathbf{L 3}$ could improve the yields slightly (Table 1, entries $2-4$ ). It was surprisingly found that bidentate ligands L5-L7, which performed well in Pd-catalyzed AAA [50,51], could not deliver any product (Table 1 , entries 5-7). Then, the solvent effect was investigated (Table 1, entries 8-13), and DCE proved to be the best choice for this reaction. An interesting thing to note is that when $\mathrm{MeOH}$ was employed as solvent, only trace product was produced (Table 1 , entries 9). This phenomenon is agreed well with Xiao's work, attributing to the hydrogen bond interactions between nitro- gen anion and protic solvent. Next, we screened different bases to improve the yield and ee. While weak bases reduced the reaction efficiency and strong bases resulted in the decomposition of starting compound 2a (Table 1, entries 14 and 15). Finally, the enantioselectivity of the product was further improved to $91 \%$ ee in a higher yield $(70 \%)$ by lowering the reaction temperature to $0^{\circ} \mathrm{C}$ (Table 1 , entry 16).

With the optimized conditions in hand, next we turned our attention to investigating the generality of Pd-catalyzed AAA (Scheme 2). Notably, all these reactions gave terminal substituted products as the main products with linear/branch ratio $>$ 20:1. In addition, extremely high $E$-selectivity ( $>20: 1$ ) was consistently secured. First, the substrate scope of $\alpha$-thiocyanato ketones $\mathbf{2}$ was examined (3ab-3al). With electronically variable functional groups on the different positions of $\alpha$-thiocyanato ketones, all the linear selectivie allylated products could be obtained in excellent enantioselectivities (90\% to $95 \%$ ee). However, it seems that the electronic effect of substituents has little impact on on the yield. For example,
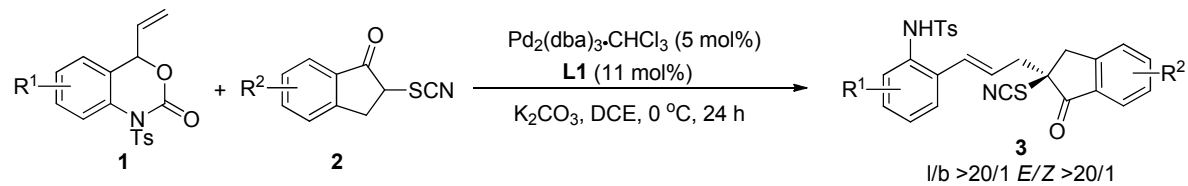

l/b $>20 / 1 E / Z>20 / 1$

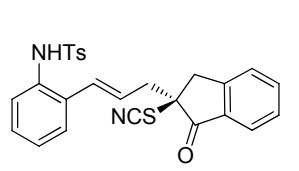

3aa: $67 \%$ yield, $91 \%$ ee

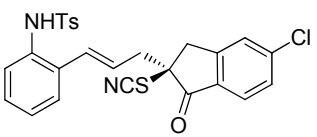

3ae: $37 \%$ yield, $92 \%$ ee NHTs

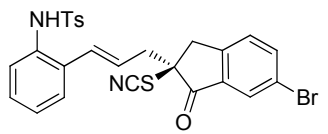

3ai: $49 \%$ yield, $91 \%$ ee

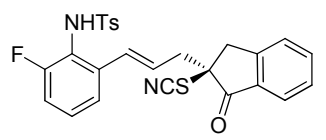

3ba: $43 \%$ yield, $93 \%$ ee NHTs

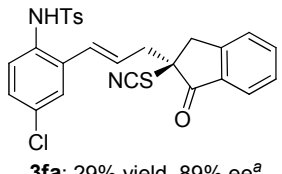

3fa: $29 \%$ yield, $89 \% \mathrm{ee}^{\mathrm{a}}$<smiles>C[As]c1cccc(F)c1/C=C/C[C@@]1(S)Cc2ccccc2C1=O</smiles>

3ja: $41 \%$ yield, $86 \%$ ee

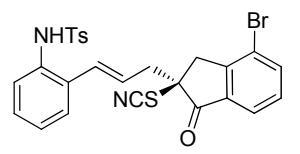

3ab: $44 \%$ yield, $90 \%$ ee NHTs

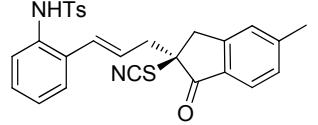

3af: $33 \%$ yield, $90 \%$ ee

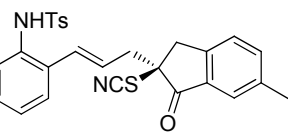

3aj: $59 \%$ yield, $93 \%$ ee

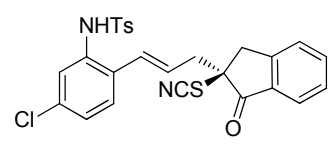

3ca: $38 \%$ yield, $94 \%$ ee $^{b}$

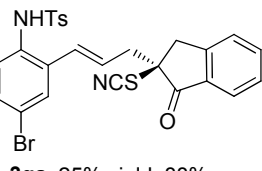

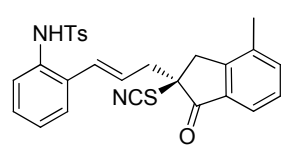

3ac: $62 \%$ yield, $90 \%$ ee<smiles>COc1ccc2c(c1)C[C@@](CC=Cc1ccccc1NC(F)(F)F)(S[Sn])C2=O</smiles>

3ag: $51 \%$ yield, $95 \%$ ee ${ }^{a}$

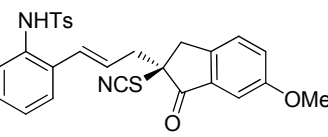

3ak: $71 \%$ yield, $90 \%$ ee

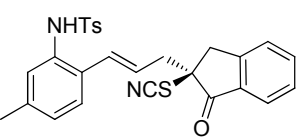

3da: $67 \%$ yield, $93 \%$ ee

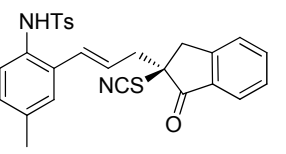

3ha: $74 \%$ yield, $90 \%$ ee

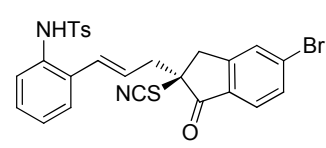

3ad: $53 \%$ yield, $90 \%$ ee

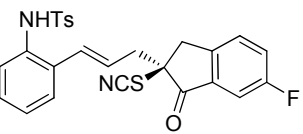

3ah: $64 \%$ yield, $90 \%$ ee

$\mathrm{NHTs}$
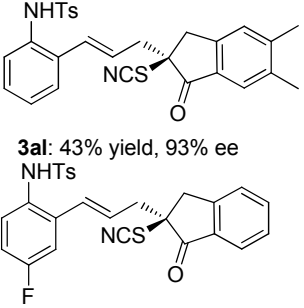

3ea: $36 \%$ yield, $88 \%$ ee

NHTs

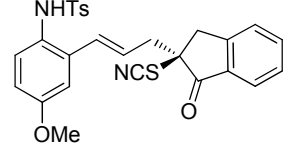

3ia: $65 \%$ yield, $92 \%$ ee

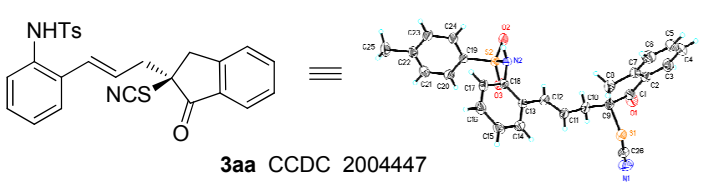

Scheme 2 Substrate scope of vinyl benzoxazinanones $\mathbf{1}$ and $\alpha$-thiocyanato ketones 2 . Reaction conditions: $\mathbf{1}$ (0.2 mmol), 2 (0.4 mmol), $\mathrm{Pd}_{2}(\mathrm{dba})_{3} \cdot \mathrm{CHCl}_{3}$ (5 mol\%), chiral ligand $\mathbf{L 1}$ (11 mol\%) and $\mathrm{K}_{2} \mathrm{CO}_{3}$ (2.0 equiv.) in $2 \mathrm{~mL} \mathrm{DCE}$ at $0{ }^{\circ} \mathrm{C}$ for 24 h, isolated yields. a 4.0 equiv. $\mathrm{K}_{2} \mathrm{CO}{ }_{3}$ was employed. 
C6-fluoro and C6-methoxy-substituted substrates generated the products with similar yields (3ah vs 3ak). While the position of the substituents exerts some influence on the yield, because C4 and C6-methyl-substituted substrates delivered the corresponding products in good yields, better than that afforded by C5-methyl-substituted $\alpha$-thiocyanato ketone (3ac and 3aj vs. 3af). Furthermore, we noticed that if C5 and C6-dimethyl-substituted substrate was used, the isolated yield was reduced moderately compared with C6-methyl-substituted substrate (3al vs. 3aj). Next, the generality of vinyl benzoxazinanones $\mathbf{1}$ was studied (3ba-3ja). A wide range of vinyl benzoxazinanones bearing electronically distinct substituents at different positions could successfully afford the linear-selective products with high enenatioselectivities (86\%-93\% ee). Compared with the electron-donating-group-functionalized arenes, substrates with electron-withdrawing groups gave the desired products with slightly reduced yields (for example, 3ea vs. 3ia). The positions of the substituents seem to impose a delicate effect on controlling the enantioselectivity. For instance, C3-fluoro-substituted substrate delivered the product $\mathbf{3 j a}$ in a slightly lower enantioselectivity than the products with functional groups on other positions. The absolute configuration of 3aa was determined by single crystal X-ray diffraction analysis (see the Supporting Information) and the stereochemistry of the other structures was assigned by analogy.

Then we conducted several experiments to get more information about this reaction (Scheme 3). Firstly, chain-like $\alpha$-SCN ketone $\mathbf{2 m}$ and six-membered $\alpha$-SCN ketone $\mathbf{2 n}$ were used as nucleophiles but no product was obtained, which meant the structure of five-membered ring may exhibit the strongest nucleophilicity. Another indanone $\mathbf{4}$ with $\alpha$-Cl substituted neither gave desired product, which indicated that thiocyanato group may have some special effect during the reaction. Finally, $\operatorname{Ir}(\mathrm{I})$ catalyst and various phosphoramidite ligands were screened for this reaction. Although the branch spiro-product $\mathbf{5}$ could be prepared, the low enantioselectivity remained a huge problem.

To probe into the mechanism of the $\operatorname{Pd}(0)$ catalyzed asymmetric terminal allylic substitution with $\alpha$-thiocyanato ketones, we carried out DFT calculations using B3LYP-D3/cc-pVDZ method (computational details are given in the SI). We chose $\mathbf{L 1}$ as the model ligand, $\mathbf{1 a}$ and $\mathbf{2 a}$ as the model substrate since

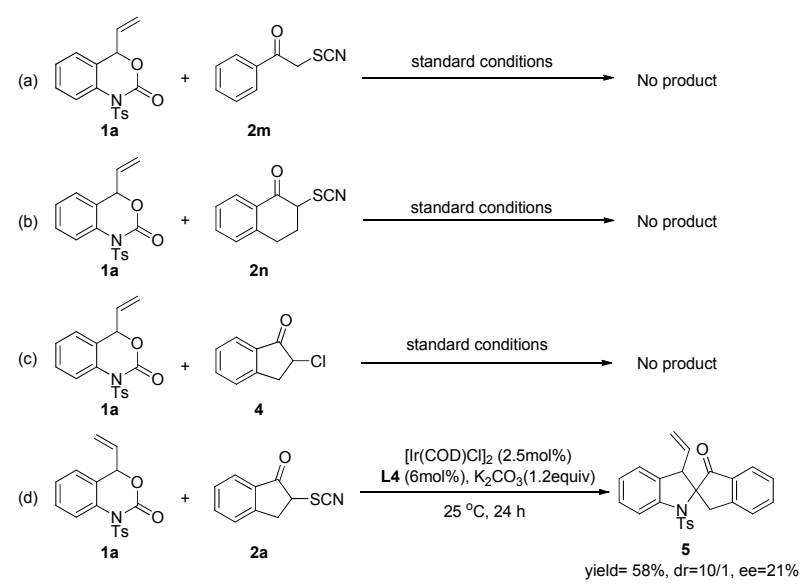

Scheme 3. Control experiments. they were found to have good yield and selectivity. 1a is assumed to detach $\mathrm{CO}_{2}$ under $\mathrm{Pd}$ catalysis to get $\operatorname{Pd}(\mathbf{L 1}) \mathbf{1} \mathbf{a}^{\prime}$, and $\mathbf{2 a}$ is expected to isomerize to its enol form $\mathbf{2} \mathbf{a}^{\prime}$ easily. Then three reaction models for the next enantioselective and regioselective C-C bond formation were proposed (Fig. 1). Model A was reported in previous literatures where amino anion functioned as a directing group through hydrogen bond effect with enolate hydroxyl group [8-12]. Therefore, we started the simulation with $\operatorname{Pd}(\mathbf{L 1}) \mathbf{1} \mathbf{a}^{\prime}$ and $\mathbf{2} \mathbf{a}^{\prime}$. It was found that the proton transferred from enolate hydroxyl of $2 \mathbf{a}^{\prime}$ to amino anion of $\operatorname{Pd}(\mathbf{L 1}) 1 a^{\prime}$ automatically and enol ion was formed, which indicated no hydrogen bond effect existed in the reaction. However, proton transfer from $\mathbf{2} \mathbf{a}^{\prime}$ to $\mathbf{1} \mathbf{a}^{\mathbf{\prime}}$ is not preferred both in gas phase $(\Delta G=+25.2 \mathrm{kcal} / \mathrm{mol})$ and in DCE solvent $(\Delta G=+8.2$ $\mathrm{kcal} / \mathrm{mol}$ ). Furthermore, Model B was considered where $\pi$-allyl $\operatorname{Pd}(\mathbf{L 1})$ and enol ion constitute an ion pair complex. Different from previous reports [52,53], monodentate phosphoramidite ligand was used and the ratio of ligand to palladium is 1.1:1, which means that palladium is not fully coordinated. This ion pair complex is quite unstable and transformed to complex 6 (Model C) easily where oxygen anion and adjacent sulfur coordinated with Pd. The calculated binding free energy of enol ion and $\pi$-allyl Pd(L1) at standard condition is up to $23.5 \mathrm{kcal} / \mathrm{mol}$, which proves model $\mathrm{C}$ is the most possible reaction pathway for the enantioselective and regioselective $\mathrm{C}-\mathrm{C}$ bond formation. This finding represents an unprecedented inner-sphere mechanism for Pd-catalyzed allylic alkylation with monodentate phosphoramidite ligand.

The mechanism of both terminal substitution reported in this work and the branch selective substitution reported before were evaluated, and the reaction profile were shown in Fig. 2. In the terminal allylic substitution to form 3aa, 6 firstly isomerize to $\mathbf{6} \mathbf{a}$ where allylic ligand coordinated monodentately. Then $\mathbf{6 a}$ undergoes allylic substitution with $\alpha$-thiocyanato ketones to reach the $\mathbf{7 a}$ which is the Pd coordinated 3aa. The rate-determined step is the allylic substitution, with the overall barrier of $18.8 \mathrm{kcal} / \mathrm{mol}$. For the comparison, we also studied the branched substitution to form $\mathbf{7 b}$. The mechanism is simi-

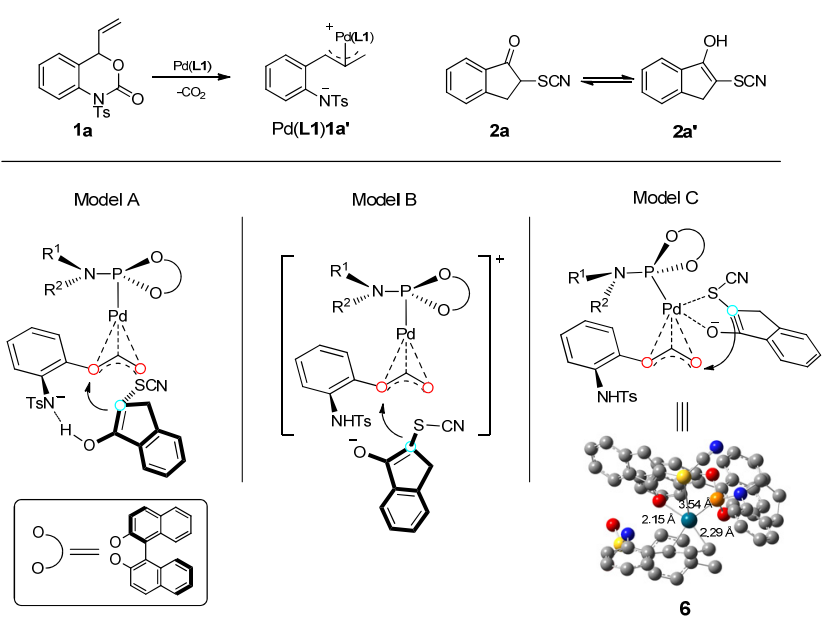

Fig. 1. Three possible reaction models proposed for this allylic alkylation reaction. Model A: hydrogen bond direction. Model B: ion pair intermediate. Model C: $\alpha$-thiocyanato ketone coordinated $\pi$-allyl $\mathrm{Pd}$ complex. 


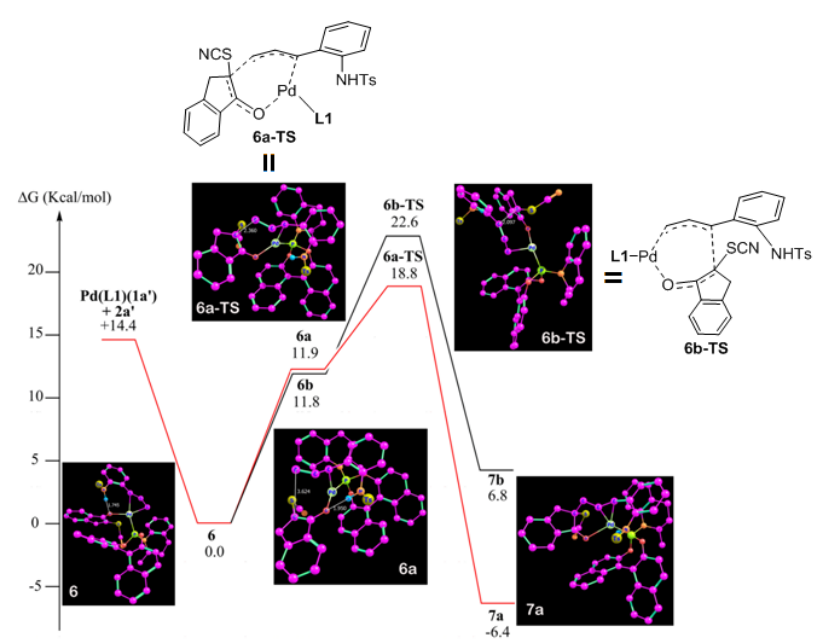

Fig. 2. The reaction energy profile for the Pd(0)-catalyzed allylic substitution with $\alpha$-thiocyanato ketones through inner-sphere mechanism. Energies are the free energies in DCE in $\mathrm{kcal} / \mathrm{mol}$, and bond lengths are in $\AA$. The hydrogens attached on carbon atoms was omitted for clarity.

lar, while the rate-determined barrier was found to be 22.6 $\mathrm{kcal} / \mathrm{mol}$. In agreement with the experimental results, the calculation also predicts that the terminal substitution is preferred. Detailed analysis shows that the higher steric repulsion from the secondary carbon in the transition state of branched substitution is the major reason for the relatively high barrier. The stereoselectivity was also studied theoretically. We found that for the terminal substitution, the rate-determined barrier to form the experimentally unobserved stereoisomer was higher by $0.7 \mathrm{kcal} / \mathrm{mol}$ in enthalpy, and $0.5 \mathrm{kcal} / \mathrm{mol}$ in free energy. The corresponding substitution product, 7c, is less stable than $7 \mathbf{a}$ by $1.9 \mathrm{kcal} / \mathrm{mol}$ which could also be one of the reasons for the observed stereoselectivity. The disfavored branched substitution has better stereoselectivity, with rate-determined barriers of $24.1 \mathrm{kcal} / \mathrm{mol}$ and $27.8 \mathrm{kcal} / \mathrm{mol}$ for the two stereoisomers. Furthermore, computational experiments suggest that the chiral carbon in the L1 ligand is important for the stereoselectivity. When we change it from $R$ to $S$, we found that the experimentally observed stereoisomer is not preferred any more.

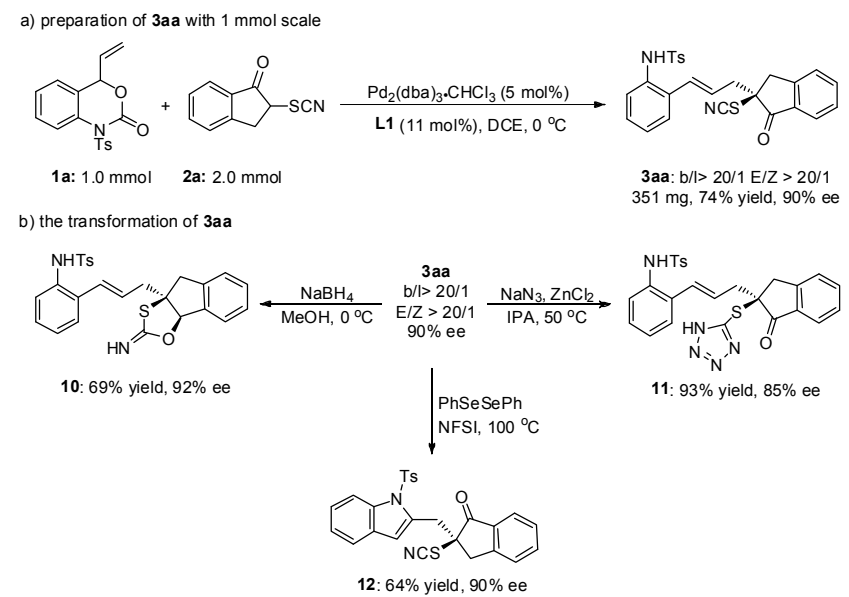

Scheme 5. Synthetic utility.

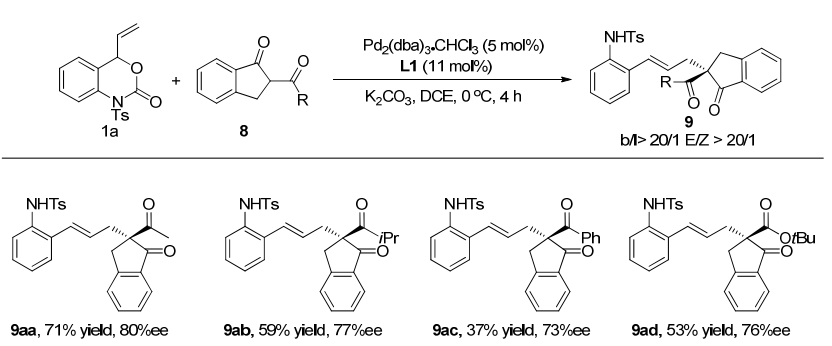

Scheme 4. Substrate scope of $\alpha$-acyl indanones. Reaction conditions: 1a ( $0.1 \mathrm{mmol}$ or $0.05 \mathrm{mmol}$ ), 8 (1.5 equiv.), $\mathrm{Pd}_{2}(\mathrm{dba})_{3} \cdot \mathrm{CHCl}_{3}(5 \mathrm{~mol} \%$ ), chiral ligand $\mathbf{L 1}(11 \mathrm{~mol} \%)$ and $\mathrm{K}_{2} \mathrm{CO}_{3}$ (2.0 equiv.) in DCE (1.0 mL or 0.5 $\mathrm{mL}$ ) at $0^{\circ} \mathrm{C}$ for $4 \mathrm{~h}$, isolated yields.

According to the proposed mechanism, nucleophiles with acidic $\mathrm{C}-\mathrm{H}$ and coordinated functional groups were predicted to undergo this reaction pathway and give linear products. As a result, $\alpha$-acyl indanones 8 were applied to examine the computational results (Scheme 4). The target products 9 were successfully obtained with moderate yield and enantioselectivity, whether R were alkyl, phenyl or alkoxy groups.

To demonstrate the synthetic utility of the Pd-catalyzed asymmetric decarboxylative reaction, 1 mmole scale synthesis of the product 3aa was conducted under standard conditions (Scheme 5(a)). To our delight, a slightly improved yield and maintained enantioselectivity were achieved. Moreover, 3aa could be transformed to useful heterocyclic compounds with simple treatments (Scheme 5(b)). Thiazolidine heterocycle $\mathbf{1 0}$ could be obtained with moderate yield and retained enantioselectivity with sodium borohydride as reductant. When processing with sodium azide and anhydrous zinc dichloride, tetrazole 11 was generated in 93\% yield and $85 \%$ ee. Compound 12 containing indole scaffold could be successfully produced with retained enantioselectivity in the presence of diphenyl diselenide.

\section{Conclusions}

In summary, we have successfully developed the Pd-catalyzed linear-selective AAA of vinyl benzoxazinanones with $\alpha$-thiocyanato ketones. This reaction features high $E$-selectivity and chemoselectivity through unique inner-sphere mechanism, which is proved by DFT studies and arises from the acidic $\mathrm{C}-\mathrm{H}$ and the slightly strong coordination effect of $\alpha$-thiocyanato ketones and $\alpha$-acyl indanones with palladium, and generates a range of thiocyanates directly attached by quaternary carbon compounds with moderate to good yields and excellent enantioselectivities. Furthermore, the products could be transformed to other useful chiral heterocycles with simple treatments, which shows a great potential in synthetic chemistry. These findings have exhibited a distinguished reaction mode of Pd-catalyzed decarboxylative reactions of vinyl benzoxazinanones and provided a new direction for future research.

\section{Electronic supporting information}

Supporting information is available in the online version of 
this article.

\section{References}

[1] C. Wang, N. Pahadi, J. A. Tunge, Tetrahedron, 2009, 65, 5102-5109.

[2] T. R. Li, Y. N. Wang, W. J. Xiao, L. Q. Lu, Tetrahedron Lett., 2018, 59, 1521-1530.

[3] X. L. Hou, Z. Yang, H. N. C. Wong, in: G. W. Gribble, T. Gilchrist, ed., Progress in Heterocyclic Chemistry, Pergamon, Oxford, 2003.

[4] A. Katritzky, C. Ramsden, J. Joule, V. Zhdankin, Handbook of Heterocyclic Chemistry, 3rd ed., Elsevier, Amsterdam, 2010.

[5] S. Eguchi, Bioactive Heterocycles II, Springer, Berlin, 2007.

[6] C. Cabrele, O. Reiser, J. Org. Chem., 2016, 81, 10109-10125.

[7] G. J. Mei, C. Y. Bian, G. H. Li, S. L. Xu, W. Q. Zheng, F. Shi, Org. Lett., 2017, 19, 3219-3222.

[8] Y. N. Wang, B. C. Wang, M. M. Zhang, X. W. Gao, T. R. Li, L. Q. Lu, W. J. Xiao, Org. Lett. 2017, 19, 4094-4097.

[9] Y. N. Wang, Q. Xiong, L. Q. Lu, Q. L. Zhang, Y. Wang, Y. Lan, W. J. Xiao, Angew. Chem. Int. Ed., 2019, 58, 11013-11017.

[10] T. R. Li, F. Tan, L. Q. Lu, Y. Wei, Y. N. Wang, Y. Y. Liu, Q. Q. Yang, J. R. Chen, D. Q. Shi, W. J. Xiao, Nat. Commun., 2014, 5, 5500.

[11] Q. Wang, X. Qi, L. Q. Lu, T. R. Li, Z. G. Yuan, K. Zhang, B. J. Li, Y. Lan, W. J. Xiao, Angew. Chem. Int. Ed., 2016, 55, 2840-2844.

[12] Q. Wang, T. R. Li, L. Q. Lu, M. M. Li, K. Zhang, W. J. Xiao, J. Am. Chem. Soc., 2016, 138, 8360-8363.

[13] C. Wang, J. A. Tunge, J. Am. Chem. Soc., 2008, 130, 8118-8119.

[14] Y. Wei, L. Q. Lu, T. R. Li, B. Feng, Q. Wang, W. J. Xiao, H. Alper, Angew. Chem. Int. Ed., 2016, 55, 2200-2204.

[15] L. A. Leth, F. Glaus, M. Meazza, L. Fu, M. K. Thogersen, E. A. Bitsch, K. A. Jorgensen, Angew. Chem. Int. Ed., 2016, 55, 15272-15276.

[16] M. M. Li, Y. Wei, J. Liu, H. W. Chen, L. Q. Lu, W. J. Xiao, J. Am. Chem. Soc., 2017, 139, 14707-14713.

[17] G. J. Mei, D. Li, G. X. Zhou, Q. Shi, Z. Cao, F. Shi, Chem. Commun., 2017, 53, 10030-10033.

[18] C. Wang, Y. Li, Y. Wu, Q. Wang, W. Shi, C. Yuan, L. Zhou, Y. Xiao, H. C. Guo, Org. Lett., 2018, 20, 2880-2883.

[19] Y. N. Lu, J. P. Lan, Y. J. Mao, Y. X. Wang, G. J. Mei, F. Shi, Chem. Commun., 2018, 54, 13527-13530.

[20] H. W. Zhao, N. N. Feng, J. M. Guo, J. Du, W. Q. Ding, L. R. Wang, X. Q. Song, J. Org. Chem., 2018, 83, 9291-9299.
[21] C. Guo, M. Fleige, D. Janssen-Muller, C. G. Daniliuc, F. Glorius, J. Am. Chem. Soc., 2016, 138, 7840-7843.

[22] C. Guo, D. Janssen-Müller, M. Fleige, A. Lerchen, C. G. Daniliuc, F. Glorius. J. Am. Chem. Soc. 2017, 139, 4443-4451.

[23] Z. S. Qi, L. H. Kong, X. W. Li, Org. Lett., 2016, 18, 4392-4395.

[24] M. Sun, X. Wan, S. J. Zhou, G. J. Mei, F. Shi, Chem. Commun., 2019, $55,1283-1286$.

[25] L. C. Akullian, M. L. Snapper, A. H. Hoveyda, Angew. Chem. Int. Ed., 2003, 42, 4244-4247.

[26] B. Liu, S. F. Zhu, W. Zhang, C. Chen, Q. L. Zhou, J. Am. Chem. Soc., 2007, 129, 5834-5835.

[27] Z. Lu, A. Wilsily, G. C. Fu, J. Am. Chem. Soc., 2011, 133, 8154-8157.

[28] J. S. Arnold, H. M. Nguyen, J. Am. Chem. Soc., 2012, 134, 8380-8383.

[29] J. W. Liu, Z. B. Han, X. M. Wang, Z. Wang, K. L. Ding, J. Am. Chem. Soc., 2015, 137, 15346-15349.

[30] B. B. Zhan, L. Wang, J. Luo, X. F. Lin, B. F. Shi. Angew. Chem. Int. Ed., 2020, 59, 3568-3572.

[31] G.-X. Jiang, B. List, Angew. Chem. Int. Ed., 2011, 50, 9471-9474.

[32] Z. L. Tao, W. Q. Zhang, D. F. Chen, A. Adele, L. Z. Gong, J. Am. Chem. Soc., 2013, 135, 9255-9258.

[33] L. Z. Lin, Y. Y. Che, P. B. Bai, C. Feng, Org. Lett,, 2019, 21, 7424-7429.

[34] Q. Q. Hang, S. J. Liu, L. Yu, T. T. Sun, Y. C. Zhang, G. J. Mei, F. Shi, Chin. J. Chem., 2020, 38, 1612-1618.

[35] L. Yu, Z. Q. Zhu, M. Sun, G. J. Mei, F. Shi, Synthesis, 2019, 51, $1655-1661$.

[36] X. Wan, M. Sun, J. Y. Wang, L. Yu, Q. Wu, Y. C. Zhang, F. Shi, Org. Chem. Front., 2020, 7, 3967-3998.

[37] F. Tian, W. L. Yang, T. Ni, J. Zhang, W. P. Deng, Sci China Chem, 2021, 64, 34-40.

[38] N. Fusetani, H. J. Wolstenholme, K. Shinoda, N. Asai, S. Matsunaga, Tetrahedron Lett., 1992, 33, 6823-6826.

[39] H. Hirota, Y. Tomono, N. Fusetani, Tetrahedron, 1996, 52, 2359-2368.

[40] H. Hirota, T.Okino, E. Yoshimura, N. Fusetani, Tetrahedron, 1998, $54,13971-13980$.

[41] E. R. A. Yasman, V. Wray, P. Proksch, J. Nat. Prod., 2003, 66, 1512-1514.

[42] M. J. Garson, J. S. Simpson, Nat. Prod. Rep., 2004, 21, 164-179.

[43] J. Emsermann, U. Kauhl, T. Opatz, Mar. Drugs, 2016, 14, 16.

[44] S. Nakamura, M. Ohara, M. Koyari, M. Hayashi, K. Hyodo, N. R.

\section{Graphical Abstract}

Chin. J. Catal., 2021, 42: 1227-1237 doi: 10.1016/S1872-2067(20)63751-2

Palladium-catalyzed enantioselective linear allylic alkylation of vinyl benzoxazinanones: An inner-sphere mechanism

Kai Wang, Binli Wang, Xianghui Liu, Hongjun Fan *, Yan Liu *, Can $\mathrm{Li}^{*}$

Dalian Institute of Chemical Physics, Chinese Academy of Sciences; University of Chinese Academy of Sciences

A unique inner-sphere mechanism for the palladium-catalyzed asymmetric allylic alkylation of vinyl benzoxazinanones characterized with extremely high linear-selectivity, $E$-selectivity and stereoselectivity.

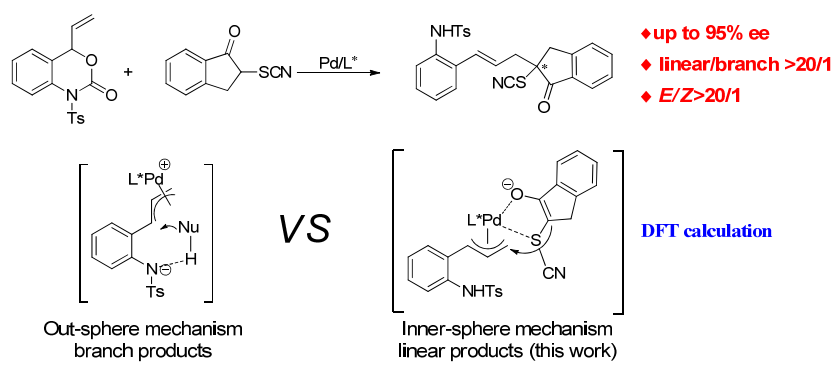


Nabisaheb, Y. Funahashi, Org. Lett., 2014, 16, 4452-4455.

[45] L. Yu, X. Y. Wu, M. J. Kim, V. Vaithiyanathan, Y. D. Liu, Y. Tan, W. L. Qin, C. E. Song, H. L. Yan. Adv. Synth. Catal., 2017, 359, 1879-1891.

[46] W. Z. Zhang, X. Wang, B. Q. Zhu, D. Zhu, J. L. Han, A. Wzorek, A. Sato, V. A. Soloshonok, J. Zhou, Y. Pan, Adv. Synth. Catal., 2017, 359, 4267-4273.

[47] X. H. Liu, K. Wang, Y. Liu, C. Li, Chem. Eur. J., 2021, 27, 735-739.

[48] W. B. Liu, C. Zheng, C. X. Zhuo, L. X. Dai, S. L. You, J. Am. Chem. Soc., 2012, 134, 4812-4821.
[49] Q. Cheng, Y. Wang, S. L. You, Angew. Chem. Int. Ed., 2016, 55, 3496-3499.

[50] W. H. Zheng, B. H. Zheng, Y. Zhang, X. L. Hou, J. Am. Chem. Soc., 2007, 129, 7718-7719.

[51] B. M. Trost, J. E. Schultz, T. W. Chang, M. R. Maduabum, J. Am. Chem. Soc., 2019, 141, 9521-9526.

[52] B. M. Trost, R. Radinov, E. M. Grenzer, J. Am. Chem. Soc., 1997, 119, 7879-7880.

[53] B. M. Trost, G. M. Schroeder, J. Am. Chem. Soc., 1999, 121, 6759-6760.

\title{
钯催化烯基苯并噁嗪酮基于内球型机理的线性和不对称烯丙基烷基化反应
}

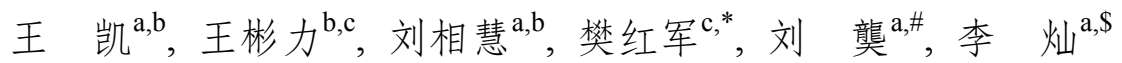 \\ a中国科学院大连化学物理研究所, 催化基础国家重点实验室, 辽宁大连 116023 \\ ${ }^{\mathrm{b}}$ 中国科学院大学, 北京 100049 \\ c中国科学院大连化学物理研究所, 分子反应动力学国家重点实验室, 辽宁大连 116023
}

摘要: 烯基苯并噁嗪酮作为底物参与反应受到有机合成工作者的广泛关注. 在过渡金属催化作用下, 烯基苯并噁嗪酮脱除 一分子二氧化碳, 生成的两性离子中间体既可以被亲核试剂进攻, 得到结构丰富的芳香胺, 也可以作为 1,4-偶极子与硫叶立 德, 缺电子烯烃或 $\alpha, \beta$-不饱和醛参与成环, 分别生成相应的五元、六元或七元含氮杂环. 后者广泛存在于农药、医药和生物 活性分子中. 已报道的钯催化烯基苯并噁嗪酮的不对称转化反应中, 支链结构是主产物, 线性结构产物比较少见. 这是因 为烯丙基邻位的氨基负离子与亲核试剂存在氢键或者静电作用, 诱导亲核试剂进攻位阻更大但是能量更低的苄位. 不同 于传统的钯催化烯丙基取代反应, 产物的结构通常是由亲核试剂的软硬程度决定. 除了化学选择性的问题, 产物中双键的 Z/E构型和立体选择性的控制也同样成为挑战. 近期Li课题组 (Org. Lett., 2016, 18, 4392-4395)基于定位基辅助的 Rh(III)催化 C-H活化策略实现了芳烃烯丙基化得到线性产物. Shi课题组(Chem. Commun., 2019, 55, 1283-1286)利用 Ir(I)和Brönsted酸协 同催化实现了吅内酯进攻端位烯丙基. 但是, 这些反应仅能得到消旋产物. 发展不对称的线性烯丙基取代反应, 不仅可以 拓展烯基苯并噁嗪酮的应用前景, 还可以合成具有手性的邻乙烯基芳香胺.

本文采用 $\alpha$-硫氰基取代的荫酩作亲核试剂, 不同于以往文献报道的机理,氨基负离子对烯醇式苑酮没有起到导向作用, 意外得到以直链为主的产物. 经过优化, 最终以联萗二酚骨架亚膦酰胺为配体, 钯作为催化剂, 成功构建了一系列与硫氧 基直接相连的季碳手性产物. 所有反应产物均有优秀的化学选择性(线性选择性> 20/1), E/Z选择性(> 20/1)和立体选择性 (最高 $95 \%$ ee). 并且该反应适用范围广泛, 基团的兼容性良好. 为解释实验结果, 本文进行相关的控制实验和DFT计算. 计 算结果表明, 由于氨基负离子碱性较强, 狮酮 $\alpha$ 位 $\mathrm{C}-\mathrm{H}$ 酸性较强, 直接发生了质子转移生成荫酮烯醇负离子, 此时氢键作用 消失不能起到导向作用. 本文还考察了狮䣳烯醇负离子与烯丙基钯通过静电作用形成离子复合物. 与之前文献报道不同, 本文采用了单齿膦配体且钯与配体等量, 这意味着钯处于配位不饱和状态, 导致离子复合物极其不稳定, 烯醇负离子与硫 氧基直接与钯配位成键, 该过程结合能高达 $23.47 \mathrm{kcal} / \mathrm{mol}$. 最终配合物中间体通过内球型机理, 经历环状过渡态得到以线 性为主的产物.

关键词: 钯催化; 不对称催化; 烯丙基烷基化; 烯基苯并噁嗪酮; 内球型机理

收稿日期: 2020-10-28. 接受日期: 2020-12-15. 上网时间: 2021-03-05.

*通讯联系人. 电话: (0411)84379913; 传真: (0411)84675584; 电子信箱: fanhj@dicp.ac.cn

\#通讯联系人. 电话: (0411)84379252; 传真: (0411)84694447; 电子信箱: yanliu503@dicp.ac.cn

\$通讯联系人. 电话: (0411)84379070; 传真: (0411)84694447; 电子信箱: canli@dicp.ac.cn

基金来源: 国家自然科学基金(21673224, 21871254, 21532006, 21472187); 大连化学物理研究所(DICP ZZBS201602); 中国科学 院战略科技先导专项(XDB17010200).

本文的电子版全文由Elsevier出版社在ScienceDirect上出版(http://www.sciencedirect.com/journal/chinese-journal-of-catalysis). 